\title{
Influence of Sumac Extract on the Physico-chemical Properties and Oxidative Stability of Some Cold Pressed Citrus Seed Oils
}

\author{
Kashif Ghafoor ${ }^{1}$, Mehmet Musa Özcan ${ }^{2 *}$, Fahad Al Juhaimi ${ }^{1}$, Elfadıl E Babiker ${ }^{1}$, and \\ Isam A. Mohamed Ahmed ${ }^{1}$ \\ ${ }^{1}$ Department of Food Science and Nutrition, College of Food and Agricultural Sciences, King Saud University, Riyadh-SAUDI ARABIA \\ ${ }^{2}$ Department of Food Engineering, Faculty of Agriculture, University of Selçuk, 42031 Konya, TURKEY
}

\begin{abstract}
The acidity values changed between $1.03 \mathrm{mgKOH} / 100 \mathrm{~g}$ (control) and $1.11 \mathrm{mgKOH} / 100 \mathrm{~g}(0.1 \%$ extract) for orange oil, $1.06 \mathrm{mgKOH} / 100 \mathrm{~g}(0.5 \%$ extract) and $1.13 \mathrm{mgKOH} / 100 \mathrm{~g}(0.1 \%$ extract) and 1.25 $\mathrm{mgKOH} / 100 \mathrm{~g}(0.5 \%$ extract) and $1.31 \mathrm{mgKOH} / 100 \mathrm{~g}$ (control) for mandarin oil. The peroxide values were determined between $1.37 \mathrm{meqO} \mathrm{O}_{2} / \mathrm{kg}(0.5 \%$ extract $)$ and $1.43 \mathrm{meqO}_{2} / \mathrm{kg}(0.1 \%$ extract $)$ for orange oil, between $1.24 \mathrm{meqO} \mathrm{O}_{2} / \mathrm{kg}$ (control) and $1.27 \mathrm{meqO}_{2} / \mathrm{kg}\left(0.1 \%\right.$ extract) for lemon and $1.60 \mathrm{meq} \mathrm{O}_{2} / \mathrm{kg}(0.5 \%$ extract) and $1.71 \mathrm{meqO}_{2} / \mathrm{kg}$ (control) in mandarin oil samples. The viscosity values of samples changed between 0.051 Pa.S (control) and $0.065 \mathrm{~Pa} . S$ (0.5\% extract) for orange, $0.051 \mathrm{~Pa} . \mathrm{S}$ (control) and $0.067 \mathrm{~Pa} . \mathrm{S}$ (0.5\% extract) lemon and $0.044 \mathrm{~Pa} . \mathrm{S}$ (control) and $0.057 \mathrm{~Pa} . \mathrm{S}(0.5 \%$ extract) in mandarin oil samples. At the end of storage study $\left(28^{\text {th }}\right.$ day), the acidity values significantly changed, and their values ranged between 2.28 $\mathrm{mgKOH} / 100 \mathrm{~g}(0.5 \%$ extract) and $3.64 \mathrm{mgKOH} / 100 \mathrm{~g}$ (control) in orange, $1.67 \mathrm{mgKOH} / 100 \mathrm{~g}(0.5 \%$ extract) and $2.28 \mathrm{mgKOH} / 100 \mathrm{~g}$ (control) in lemon and $1.74 \mathrm{mgKOH} / 100 \mathrm{~g}(0.5 \%$ extract) and $2.36 \mathrm{mgKOH} / 100 \mathrm{~g}$ (control) in mandarin oil samples. While peroxide values vary between $11.68 \mathrm{meq} \mathrm{O}_{2} / \mathrm{kg}(0.5 \%$ extract) and $32.57 \mathrm{meqO}_{2} / \mathrm{kg}$ (control) for orange, $12.55 \mathrm{meqO}_{2} / \mathrm{kg}(0.5 \%$ extract) and $34.63 \mathrm{meqO} / \mathrm{Kg}$ (control) for lemon and between $17.56 \mathrm{meqO}_{2} / \mathrm{kg}\left(0.5 \%\right.$ extract) and $37.81 \mathrm{meqO}_{2} / \mathrm{kg}$ (control) for mandarin oils, viscosity values after 28 day storage changed between $0.123 \mathrm{~Pa} . \mathrm{S}(0.5 \%$ extract) and $0.675 \mathrm{~Pa} . \mathrm{S}$ (control) in orange, 0.257 Pa.S (0.5\% extract) and 0.697 Pa.S (control) in lemon and $0.215 \mathrm{~Pa} . \mathrm{S}(0.5 \%$ extract) and 0.728 Pa.S (control) in mandarin oil samples.
\end{abstract}

Key words: sumac extract, citrus oils, oxidative stability, viscosity, storage, cold press

\section{Introduction}

Sumac (Rhus coriaria L.) is traditionally being used as a natural medication. The use of the plant in seasonings and flavoring agents has been the mainstay of indigenous remedies in different dietary cultures around the globe ${ }^{1)}$. Sumac is used as a spice, and has been used in cooking for millennia. About 2000 years ago, the Greek physician Pedenius Dioscorides wrote in his voluminous "De Materia Medica" about the healthful properties of sumac, principally as a diuretic and anti-flatulent ${ }^{2}$. The leave, stick and seeds of sumac plant contain substantial amounts of several biologically important components, and the most important of those are the phenolic compounds ${ }^{1,3-8)}$. Sumac is one of the major sources of phenolic, bioactive phytochemical compound and its functional ingredients have widespread and potential usability in chemical and pharmaceutical industries $^{9)}$. Candan and Sökmen ${ }^{10)}$ reported that antioxidative capacity of $R$. coriaria fruits has been evaluated using in vitro systems. The $\mathrm{IC}_{50}$ value of the sumac extracts, for lipid peroxidation inhibition, was estimated as $1200 \mu \mathrm{g} / \mathrm{mL}$ in the $\mathrm{Fe}^{2+}$-ascorbate system ${ }^{10)}$. A previous study about stabilizing food products using sumac extracts included the use of a methanolic extract of the fruits added to sunflower oil stored at $70^{\circ} \mathrm{C}$ and peroxide values of the stored oil was measured at regular intervals. Sumac extract was found to be very effective in stabilizing the sunflower oil ${ }^{11}$. Antioxidant properties for stabilizing peanut was also reported in which methanol extracts of $R$. coriaria fruits and leaves were added to peanut oil at concentration of 1 to $5 \%(\mathrm{w} / \mathrm{v})$ and it was observed that the formation of hydroperoxide

\footnotetext{
*Correspondence to: Mehmet Musa Özcan, Department of Food Engineering, Faculty of Agriculture, University of Selçuk, 42031 Konya, TURKEY

E-mail: mozcan@selcuk.edu.tr

Accepted January 21, 2020 (received for review November 17, 2019)

Journal of Oleo Science ISSN 1345-8957 print / ISSN 1347-3352 online

http://www.jstage.jst.go.jp/browse/jos/ http://mc.manusriptcentral.com/jjocs
} 
was inhibited during the initial 7 day storage, however after 28 day storage, the antioxidant potential of sumac extract substantially decreased in comparison to BHA controls ${ }^{3)}$. Ferk et $a l .{ }^{12)}$ estimated the antioxidant effect of sumac to be 50 fold more than vitamin $\mathrm{C}$ and $\mathrm{E}$. Antioxidant capacity and radical scavenging activity of sumac water extract were significantly higher than that of ethanol extract ${ }^{13)}$. Sumac water extract was more effective than BHT, and could be added to meat products (sausage) to enhance the overall product quality ${ }^{14)}$. The aim of current study was to investigate the effect of sumac extracts on physico-chemical properties and oxidative stability of cold pressed orange, lemon and mandarin seed oils.

\section{Material and Methods}

\subsection{Material}

In this study, orange (Barile cv.), lemon (Interdonato cv.) and mandarin (Clementine cv.) cultivated in Adana and Mersin provinces in Turkey were used to obtain seed oil. Seed from these fruits were separated from pulp manually, and washed with distilled water and dried at room temperature. The dried kernels were milled in laboratory to pass through 60-mesh sieve, and the samples were kept at $4^{\circ} \mathrm{C}$ before further processing and analyses. Ripened Sumac (Rhus coriaria L.) fruits were collected from Mersin (Gülnar-Büyükeceli) province in Turkey during September 2018 and dried at room temperature. They were ground to a powdered form using a lab scale grinder.

\subsection{Method}

\subsubsection{Cold press}

Citrus seed oils from orange, lemon and mandarin were obtained using cold screw extraction process at room temperature, where the initial rotation was $1500 \mathrm{rpm}$ and the final one was approximately $3600 \mathrm{rpm}$. After broken or damaged citrus seeds and other impurities such as stem and skin were removed, whole seeds were extracted with cold screw press (2-6 liter/h capacity) without heat treatment. The impurities were removed to ensure high quality oil. After pressing, the oil was purified from solid impurities by sedimentation for one week. Sedimentation process followed filtration and storage of the purified oil in hermetically closed colored bottle under the nitrogen at $-18^{\circ} \mathrm{C}$.

\subsubsection{Extraction from sumac fruit}

About 10g of ground sumac (Rhus coriaria L.) sample was extracted using Soxhlet apparatus for $5 \mathrm{~h}$ and methanol was used as solvent. The extracts were filtered using filter paper, and the solvent was removed using rotary evaporator $\left(40^{\circ} \mathrm{C}+\right.$ Vacuum $)$. In experiment, three different levels $(0.1 \%, 0.3 \%$ and $0.5 \%)$ of sumac extracts were used as additives to three different citrus seed oils. Sumac extracts at a concentration of $0.1 \%, 0.3 \%$ and $0.5 \%$ were added into each $50 \mathrm{~mL}$ citrus oil in three petri plates, respectively. Then the oil in the petri plate was thoroughly mixed and kept at $50^{\circ} \mathrm{C}$ for 28 days. Analyzes were carried out at weekly intervals.

2.2.3 Analysis

The acid value, iodine value, peroxide value, saponification value, unsaponifiable matter, density, refractive index, and viscosity of citrus seed oils were determined using standard methods ${ }^{15)}$.

\subsubsection{Statistical analyses}

The analysis of variance (ANOVA) was performed by using JMP version 9.0 (SAS Inst. Inc., Cary, N.C.U.S.A). All analyses were carried out three times and the results are mean \pm standard deviation (MSTAT C) of citrus oils and sumac extract concentrations ${ }^{16)}$.

\section{Results and Discussion}

The physico-chemical properties of citrus oils treated with sumac fruit extracts are presented in Table 1. The results showed differences depending on extract concentrations and storage periods of citrus oils. Generally, acidity, peroxide values, refractive index, saponification values of treated oil samples decreased with increase of extract concentration at the beginning of storage. While acidity values change between $1.03 \mathrm{mgKOH} / 100 \mathrm{~g}$ (control) and $1.11 \mathrm{mgKOH} / 100 \mathrm{~g}(0.1 \%$ extract $)$ in case of orange oil, $1.06 \mathrm{mgKOH} / 100 \mathrm{~g}(0.5 \%$ extract $)$ and $1.13 \mathrm{mgKOH} / 100 \mathrm{~g}$ $(0.1 \%$ extract $)$ in case of lemon oil, $1.25 \mathrm{mgKOH} / 100 \mathrm{~g}$ ( $0.5 \%$ extract) and $1.31 \mathrm{mgKOH} / 100 \mathrm{~g}$ (control) in mandarin oil, peroxide values varied between $1.37 \mathrm{meqO}_{2} / \mathrm{kg}(0.5 \%$ extract $)$ and $1.43 \mathrm{meqO}_{2} / \mathrm{kg}(0.1 \%$ extract $)$ in case of orange oil, $1.24 \mathrm{meqO}_{2} / \mathrm{kg}$ (control) and $1.27 \mathrm{meqO}_{2} / \mathrm{kg}(0.1 \%$ extract) in lemon oil and $1.60 \mathrm{meqO}_{2} / \mathrm{kg}(0.5 \%$ extract $)$ and $1.71 \mathrm{meqO}_{2} / \mathrm{kg}$ (control) in mandarin oil at the beginning of storage. Depending on extract concentrations, density and viscosity values of oil samples increased. The density values were between $0.884 \mathrm{mg} / \mathrm{mL}$ (control) and 0.899 ( $0.5 \%$ extract) in orange oil, 0.857 (control) and $0.868 \mathrm{mg} /$ $\mathrm{mL}(0.5 \%$ extract) in lemon oil and $0.876 \mathrm{mg} / \mathrm{mL}$ (control) and $0.886 \mathrm{mg} / \mathrm{mL}(0.5 \%$ extract $)$ in mandarin oil. The viscosity values ranged between $0.051 \mathrm{~Pa} . \mathrm{S}$ (control) and 0.065 Pa.S (0.5\% extract) in orange seed oil, 0.051 Pa.S (control) and $0.067 \mathrm{~Pa} . \mathrm{S}(0.5 \%$ extract) in lemon seed oil and 0.044 Pa.S (control) and 0.057 Pa.S (0.5\% extract) in mandarin seed oil.

Physico-chemical properties of citrus oils treated with different sumac extract concentrations showed remarkable changes after $7^{\text {th }}$ day of storage. The acidity values were between $1.18 \mathrm{mgKOH} / 100 \mathrm{~g}(0.5 \%$ extract $)$ and 1.38 $\mathrm{mgKOH} / 100 \mathrm{~g}$ (control) for treated orange oil, 1.38 $\mathrm{mgKOH} / 100 \mathrm{~g}(0.5 \%$ extract $)$ and $1.48 \mathrm{mgKOH} / 100 \mathrm{~g}(0.1 \%$ extract) lemon oil and $1.47 \mathrm{mgKOH} / 100 \mathrm{~g}(0.5 \%$ extract) 
Table 1 Effect of sumac extract addition at different concentrations (0.1-0.5\%) on physico-chemical properties of cold pressed orange, lemon and mandarin oils during storage.

\begin{tabular}{|c|c|c|c|c|c|c|c|c|c|c|c|c|}
\hline \multirow{2}{*}{$\begin{array}{c}\text { Storage time }\left(0^{\text {th }} \text { day }\right) \\
\text { Parameter }\end{array}$} & \multicolumn{4}{|c|}{ Orange (Barile) oil with sumac extract } & \multicolumn{4}{|c|}{ Lemon (Interdonato) oil with sumac extract } & \multicolumn{4}{|c|}{ Mandarin (Clementine) oil with sumac extract } \\
\hline & Control & $0.1 \%$ & $0.3 \%$ & $0.5 \%$ & Control & $0.1 \%$ & $0.3 \%$ & $0.5 \%$ & Control & $0.1 \%$ & $0.3 \%$ & $0.5 \%$ \\
\hline $\begin{array}{l}\text { Acidity } \\
(\mathrm{mgKOH} / 100 \mathrm{~g})\end{array}$ & $\begin{array}{l}1.03 \pm \\
0.13 * \mathrm{~d}\end{array}$ & $\begin{array}{c}1.11 \pm \\
0.09 \mathrm{a}^{* *}\end{array}$ & $\begin{array}{l}1.09 \pm \\
0.07 \mathrm{~b}\end{array}$ & $\begin{array}{l}1.05 \pm \\
0.011 \mathrm{c}\end{array}$ & $\begin{array}{r}1.07 \pm \\
0.21 \mathrm{c}\end{array}$ & $\begin{array}{r}1.13 \pm \\
0.09 \mathrm{~b}\end{array}$ & $\begin{array}{r}1.15 \pm \\
0.07 \mathrm{a}\end{array}$ & $\begin{array}{r}1.06 \pm \\
0.15 \mathrm{c}\end{array}$ & $\begin{array}{r}1.31 \pm \\
0.05 \mathrm{c}\end{array}$ & $\begin{array}{r}1.37 \pm \\
0.03 \mathrm{a}\end{array}$ & $\begin{array}{r}1.33 \pm \\
0.18 \mathrm{~b}\end{array}$ & $\begin{array}{r}1.25 \pm \\
0.21 \mathrm{~d}\end{array}$ \\
\hline $\begin{array}{l}\text { Peroxide value } \\
\left(\mathrm{meqO}_{2} / \mathrm{kg}\right)\end{array}$ & $\begin{array}{r}1.38 \pm \\
0.18 \mathrm{~b}\end{array}$ & $\begin{array}{r}1.43 \pm \\
0.13 \mathrm{a}\end{array}$ & $\begin{array}{r}1.39 \pm \\
0.15 b\end{array}$ & $\begin{array}{r}1.37 \pm \\
0.18 \mathrm{c}\end{array}$ & $\begin{array}{l}1.24 \pm \\
0.09 \mathrm{~cd}\end{array}$ & $\begin{array}{r}1.27 \pm \\
0.07 \mathrm{a}\end{array}$ & $\begin{array}{r}1.25 \pm \\
0.11 \mathrm{c}\end{array}$ & $\begin{array}{l}1.26 \pm \\
0.13 \mathrm{ab}\end{array}$ & $\begin{array}{r}1.71 \pm \\
0.03 \mathrm{a}\end{array}$ & $\begin{array}{r}1.66 \pm \\
0.18 b\end{array}$ & $\begin{array}{r}1.65 \pm \\
0.11 \mathrm{c}\end{array}$ & $\begin{array}{r}1.60 \pm \\
0.08 \mathrm{~d}\end{array}$ \\
\hline $\begin{array}{l}\text { Refractive Index } \\
\left(n^{20} \mathrm{D}\right)\end{array}$ & $\begin{array}{c}1.541 \pm \\
0.004 \mathrm{a}\end{array}$ & $\begin{array}{r}1.537 \pm \\
0.009 b\end{array}$ & $\begin{array}{r}1.511 \pm \\
0.011 \mathrm{c}\end{array}$ & $\begin{array}{r}1.503 \pm \\
0.013 \mathrm{~d}\end{array}$ & $\begin{array}{r}1.514 \pm \\
0.003 \mathrm{a}\end{array}$ & $\begin{array}{l}1.512 \pm \\
0.008 \mathrm{ab}\end{array}$ & $\begin{array}{r}1.509 \pm \\
0.007 \mathrm{c}\end{array}$ & $\begin{array}{r}1.501 \pm \\
0.003 \mathrm{~d}\end{array}$ & $\begin{array}{r}1.491 \pm \\
0.005 \mathrm{a}\end{array}$ & $\begin{array}{r}1.487 \pm \\
0.003 b\end{array}$ & $\begin{array}{r}1.481 \pm \\
0.005 \mathrm{c}\end{array}$ & $\begin{array}{r}1.478 \pm \\
0.003 \mathrm{~d}\end{array}$ \\
\hline $\begin{array}{l}\text { Iodine value } \\
\left(\mathrm{gI}_{2} / 100 \mathrm{~g}\right)\end{array}$ & $\begin{array}{c}98.74 \pm \\
0.17 b\end{array}$ & $\begin{array}{c}98.81 \pm \\
1.26 \mathrm{a}\end{array}$ & $\begin{array}{c}98.69 \pm \\
1.13 \mathrm{c}\end{array}$ & $\begin{array}{r}98.67 \pm \\
1.32 \mathrm{~cd}\end{array}$ & $\begin{array}{c}111.73 \pm \\
0.28 \mathrm{c}\end{array}$ & $\begin{array}{c}112.13 \pm \\
0.29 \mathrm{a}\end{array}$ & $\begin{array}{c}111.91 \pm \\
1.13 b\end{array}$ & $\begin{array}{c}111.31 \pm \\
0.98 \mathrm{~d}\end{array}$ & $\begin{array}{c}105.71 \pm \\
0.56 \mathrm{a}\end{array}$ & $\begin{array}{c}105.32 \pm \\
0.62 b\end{array}$ & $\begin{array}{c}104.61 \pm \\
0.45 \mathrm{c}\end{array}$ & $\begin{array}{c}104.17 \pm \\
0.13 \mathrm{~d}\end{array}$ \\
\hline $\begin{array}{l}\text { Saponification value } \\
(\mathrm{mgKOH} / 100 \mathrm{~g})\end{array}$ & $\begin{array}{c}193.8 \pm \\
1.45 \mathrm{a}\end{array}$ & $\begin{array}{c}193.4 \pm \\
1.57 \mathrm{a}\end{array}$ & $\begin{array}{c}192.9 \pm \\
1.63 \mathrm{c}\end{array}$ & $\begin{array}{c}194.1 \pm \\
1.87 \mathrm{a}\end{array}$ & $\begin{array}{c}194.81 \pm \\
1.33 \mathrm{a}\end{array}$ & $\begin{array}{c}194.12 \pm \\
1.42 \mathrm{a}\end{array}$ & $\begin{array}{c}193.83 \pm \\
1.38 b\end{array}$ & $\begin{array}{c}193.24 \pm \\
1.25 \mathrm{~b}\end{array}$ & $\begin{array}{c}193.52 \pm \\
1.53 \mathrm{a}\end{array}$ & $\begin{array}{c}193.11 \pm \\
1.73 b\end{array}$ & $\begin{array}{c}192.87 \pm \\
1.27 \mathrm{c}\end{array}$ & $\begin{array}{c}192.64 \pm \\
1.19 \mathrm{~d}\end{array}$ \\
\hline $\begin{array}{l}\text { Unsaponifiable matter } \\
(\%)\end{array}$ & $\begin{array}{r}1.71 \pm \\
0.13 b\end{array}$ & $\begin{array}{r}1.74 \pm \\
0.09 \mathrm{a}\end{array}$ & $\begin{array}{r}1.70 \pm \\
0.15 \mathrm{c}\end{array}$ & $\begin{array}{r}1.69 \pm \\
0.11 \mathrm{~d}\end{array}$ & $\begin{array}{r}1.83 \pm \\
0.13 \mathrm{a}\end{array}$ & $\begin{array}{r}1.80 \pm \\
0.27 b\end{array}$ & $\begin{array}{r}1.78 \pm \\
0.23 \mathrm{c}\end{array}$ & $\begin{array}{l}1.77 \pm \\
0.09 \mathrm{~cd}\end{array}$ & $\begin{array}{r}1.19 \pm \\
0.07 \mathrm{a}\end{array}$ & $\begin{array}{r}1.17 \pm \\
0.32 b\end{array}$ & $\begin{array}{r}1.13 \pm \\
0.14 \mathrm{c}\end{array}$ & $\begin{array}{r}1.10 \pm \\
0.07 \mathrm{~d}\end{array}$ \\
\hline $\begin{array}{l}\text { Density } \\
\left(\mathrm{mg} / \mathrm{mL} ; 25^{\circ} \mathrm{C}\right)\end{array}$ & $\begin{array}{r}0.884 \pm \\
0.003 d\end{array}$ & $\begin{array}{r}0.889 \pm \\
0.001 \mathrm{c}\end{array}$ & $\begin{array}{r}0.893 \pm \\
0.005 b\end{array}$ & $\begin{array}{r}0.899 \pm \\
0.003 \mathrm{a}\end{array}$ & $\begin{array}{r}0.857 \pm \\
0.001 \mathrm{~d}\end{array}$ & $\begin{array}{r}0.859 \pm \\
0.003 \mathrm{c}\end{array}$ & $\begin{array}{r}0.863 \pm \\
0.002 b\end{array}$ & $\begin{array}{r}0.868 \pm \\
0.001 \mathrm{a}\end{array}$ & $\begin{array}{r}0.876 \pm \\
0.002 \mathrm{~d}\end{array}$ & $\begin{array}{r}0.879 \pm \\
0.002 \mathrm{c}\end{array}$ & $\begin{array}{r}0.883 \pm \\
0.001 b\end{array}$ & $\begin{array}{r}0.886 \pm \\
0.003 \mathrm{a}\end{array}$ \\
\hline $\begin{array}{l}\text { Viscosity } \\
\left(\mathrm{Pa} . \mathrm{S} ; 25^{\circ} \mathrm{C}\right)\end{array}$ & $\begin{array}{r}0.051 \pm \\
0.003 \mathrm{~d}\end{array}$ & $\begin{array}{r}0.057 \pm \\
0.005 \mathrm{c}\end{array}$ & $\begin{array}{c}0.061 \pm \\
0.007 b\end{array}$ & $\begin{array}{r}0.065 \pm \\
0.003 \mathrm{a}\end{array}$ & $\begin{array}{r}0.051 \pm \\
0.003 \mathrm{~d}\end{array}$ & $\begin{array}{c}0.059 \pm \\
0.005 \mathrm{c}\end{array}$ & $\begin{array}{r}0.064 \pm \\
0.009 \mathrm{~b}\end{array}$ & $\begin{array}{r}0.067 \pm \\
0.005 \mathrm{a}\end{array}$ & $\begin{array}{r}0.044 \pm \\
0.001 \mathrm{~d}\end{array}$ & $\begin{array}{r}0.047 \pm \\
0.003 \mathrm{c}\end{array}$ & $\begin{array}{c}0.051 \pm \\
0.007 \mathrm{~b}\end{array}$ & $\begin{array}{r}0.057 \pm \\
0.003 \mathrm{a}\end{array}$ \\
\hline Storage time ( $7^{\text {th }}$ day) & Control & $0.1 \%$ & $0.3 \%$ & $0.5 \%$ & Control & $0.1 \%$ & $0.3 \%$ & $0.5 \%$ & Control & $0.1 \%$ & $0.3 \%$ & $0.5 \%$ \\
\hline $\begin{array}{l}\text { Acidity } \\
(\mathrm{mgKOH} / 100 \mathrm{~g})\end{array}$ & $\begin{array}{r}1.38 \pm \\
0.09 \mathrm{a}\end{array}$ & $\begin{array}{r}1.29 \pm \\
0.07 \mathrm{~b}\end{array}$ & $\begin{array}{r}1.21 \pm \\
0.01 \mathrm{c}\end{array}$ & $\begin{array}{r}1.18 \pm \\
0.03 \mathrm{~d}\end{array}$ & $\begin{array}{c}1.41 \pm \\
0.05 \mathrm{c}\end{array}$ & $\begin{array}{r}1.48 \pm \\
0.01 \mathrm{a}\end{array}$ & $\begin{array}{r}1.45 \pm \\
0.11 \mathrm{~b}\end{array}$ & $\begin{array}{r}1.38 \pm \\
0.05 \mathrm{~d}\end{array}$ & $\begin{array}{r}1.59 \pm \\
0.03 b\end{array}$ & $\begin{array}{r}0.63 \pm \\
0.01 \mathrm{a}\end{array}$ & $\begin{array}{r}1.55 \pm \\
0.07 \mathrm{c}\end{array}$ & $\begin{array}{r}1.47 \pm \\
0.03 \mathrm{~d}\end{array}$ \\
\hline $\begin{array}{l}\text { Peroxide value } \\
\left(\mathrm{meqO}_{2} / \mathrm{kg}\right)\end{array}$ & $\begin{array}{r}5.27 \pm \\
0.16 \mathrm{a}\end{array}$ & $\begin{array}{r}4.93 \pm \\
0.18 \mathrm{~b}\end{array}$ & $\begin{array}{r}2.71 \pm \\
0.09 \mathrm{c}\end{array}$ & $\begin{array}{l}2.63 \pm \\
0.11 \mathrm{~cd}\end{array}$ & $\begin{array}{r}6.35 \pm \\
0.07 \mathrm{a}\end{array}$ & $\begin{array}{r}5.58 \pm \\
0.09 \mathrm{~b}\end{array}$ & $\begin{array}{r}5.33 \pm \\
0.13 \mathrm{c}\end{array}$ & $\begin{array}{r}4.61 \pm \\
0.17 \mathrm{~d}\end{array}$ & $\begin{array}{r}7.98 \pm \\
0.21 \mathrm{a}\end{array}$ & $\begin{array}{r}6.37 \pm \\
0.15 b\end{array}$ & $\begin{array}{r}5.89 \pm \\
0.11 \mathrm{c}\end{array}$ & $\begin{array}{r}5.07 \pm \\
0.13 \mathrm{~d}\end{array}$ \\
\hline $\begin{array}{l}\text { Refractive Index } \\
\left(\mathrm{n}^{20} \mathrm{D}\right)\end{array}$ & $\begin{array}{c}1.540 \pm \\
0.003 \mathrm{a}\end{array}$ & $\begin{array}{r}1.532 \pm \\
0.001 \mathrm{c}\end{array}$ & $\begin{array}{r}1.520 \pm \\
0.001 \mathrm{~d}\end{array}$ & $\begin{array}{r}1.538 \pm \\
0.005 \mathrm{~b}\end{array}$ & $\begin{array}{c}1.511 \pm \\
0.003 \mathrm{a}\end{array}$ & $\begin{array}{l}1.509 \pm \\
0.001 \mathrm{bc}\end{array}$ & $\begin{array}{l}1.510 \pm \\
0.003 \mathrm{ab}\end{array}$ & $\begin{array}{l}1.508 \pm \\
0.001 \mathrm{bc}\end{array}$ & $\begin{array}{r}1.490 \pm \\
0.003 b\end{array}$ & $\begin{array}{r}1.495 \pm \\
0.003 \mathrm{a}\end{array}$ & $\begin{array}{r}1.490 \pm \\
0.001 \mathrm{~b}\end{array}$ & $\begin{array}{r}1.482 \pm \\
0.005 \mathrm{c}\end{array}$ \\
\hline $\begin{array}{l}\text { Iodine value } \\
\left(\mathrm{gI}_{2} / 100 \mathrm{~g}\right)\end{array}$ & $\begin{array}{r}98.60 \pm \\
1.21 \mathrm{ab}\end{array}$ & $\begin{array}{c}98.73 \pm \\
1.17 \mathrm{a}\end{array}$ & $\begin{array}{c}98.57 \pm \\
1.28 \mathrm{~b}\end{array}$ & $\begin{array}{r}98.61 \pm \\
1.31 \mathrm{ab}\end{array}$ & $\begin{array}{c}111.61 \pm \\
1.46 \mathrm{~b}\end{array}$ & $\begin{array}{c}111.69 \pm \\
1.57 \mathrm{a}\end{array}$ & $\begin{array}{c}111.67 \pm \\
1.84 \mathrm{ab}\end{array}$ & $\begin{array}{c}111.20 \pm \\
1.43 \mathrm{c}\end{array}$ & $\begin{array}{c}105.21 \pm \\
1.32 \mathrm{a}\end{array}$ & $\begin{array}{c}105.18 \pm \\
1.57 \mathrm{~b}\end{array}$ & $\begin{array}{c}105.13 \pm \\
1.78 \mathrm{c}\end{array}$ & $\begin{array}{c}105.17 \pm \\
1.64 \mathrm{bc}\end{array}$ \\
\hline $\begin{array}{l}\text { Saponification value } \\
(\mathrm{mgKOH} / 100 \mathrm{~g})\end{array}$ & $\begin{array}{c}193.51 \pm \\
1.67 \mathrm{a}\end{array}$ & $\begin{array}{c}193.14 \pm \\
0.98 \mathrm{~b}\end{array}$ & $\begin{array}{c}192.97 \pm \\
0.78 \mathrm{c}\end{array}$ & $\begin{array}{c}192.86 \pm \\
1.32 \mathrm{~d}\end{array}$ & $\begin{array}{c}194.50 \pm \\
1.23 \mathrm{a}\end{array}$ & $\begin{array}{c}194.32 \pm \\
1.56 \mathrm{c}\end{array}$ & $\begin{array}{c}194.15 \pm \\
1.87 \mathrm{~d}\end{array}$ & $\begin{array}{c}194.42 \pm \\
1.36 \mathrm{a}\end{array}$ & $\begin{array}{c}193.29 \pm \\
1.54 \mathrm{a}\end{array}$ & $\begin{array}{c}193.07 \pm \\
0.87 b\end{array}$ & $\begin{array}{c}192.96 \pm \\
0.65 \mathrm{c}\end{array}$ & $\begin{array}{c}192.95 \pm \\
0.98 \mathrm{~cd}\end{array}$ \\
\hline $\begin{array}{l}\text { Unsaponifiable matter } \\
(\%)\end{array}$ & $\begin{array}{r}1.70 \pm \\
0.34 \mathrm{a}\end{array}$ & $\begin{array}{r}1.69 \pm \\
0.36 b\end{array}$ & $\begin{array}{r}1.68 \pm \\
0.45 \mathrm{c}\end{array}$ & $\begin{array}{r}1.67 \pm \\
0.33 \mathrm{~d}\end{array}$ & $\begin{array}{r}1.80 \pm \\
0.27 b\end{array}$ & $\begin{array}{r}1.79 \pm \\
0.13 \mathrm{c}\end{array}$ & $\begin{array}{r}1.77 \pm \\
0.23 \mathrm{~d}\end{array}$ & $\begin{array}{r}1.81 \pm \\
0.36 \mathrm{a}\end{array}$ & $\begin{array}{r}1.17 \pm \\
0.45 \mathrm{a}\end{array}$ & $\begin{array}{r}1.15 \pm \\
0.09 \mathrm{~b}\end{array}$ & $\begin{array}{l}1.13 \pm \\
0.11 b c\end{array}$ & $\begin{array}{l}1.16 \pm \\
0.07 \mathrm{ab}\end{array}$ \\
\hline $\begin{array}{l}\text { Density } \\
\left(\mathrm{mg} / \mathrm{mL} ; 25^{\circ} \mathrm{C}\right)\end{array}$ & $\begin{array}{r}0.883 \pm \\
0.001 \mathrm{~d}\end{array}$ & $\begin{array}{r}0.887 \pm \\
0.003 \mathrm{c}\end{array}$ & $\begin{array}{r}0.893 \pm \\
0.001 \mathrm{~b}\end{array}$ & $\begin{array}{r}0.899 \pm \\
0.005 \mathrm{a}\end{array}$ & $\begin{array}{r}0.855 \pm \\
0.003 \mathrm{~d}\end{array}$ & $\begin{array}{r}0.861 \pm \\
0.001 \mathrm{c}\end{array}$ & $\begin{array}{r}0.869 \pm \\
0.002 b\end{array}$ & $\begin{array}{r}0.875 \pm \\
0.001 \mathrm{a}\end{array}$ & $\begin{array}{r}0.875 \pm \\
0.001 \mathrm{~d}\end{array}$ & $\begin{array}{r}0.879 \pm \\
0.003 \mathrm{c}\end{array}$ & $\begin{array}{r}0.885 \pm \\
0.001 \mathrm{~b}\end{array}$ & $\begin{array}{r}0.892 \pm \\
0.003 \mathrm{a}\end{array}$ \\
\hline $\begin{array}{l}\text { Viscosity } \\
\left(\mathrm{Pa} . \mathrm{S} ; 25^{\circ} \mathrm{C}\right)\end{array}$ & $\begin{array}{c}0.093 \pm \\
0.001 \mathrm{a}\end{array}$ & $\begin{array}{r}0.104 \pm \\
0.005 \mathrm{~b}\end{array}$ & $\begin{array}{c}0.109 \pm \\
0.007 \mathrm{c}\end{array}$ & $\begin{array}{r}0.103 \pm \\
0.003 d\end{array}$ & $\begin{array}{r}0.105 \pm \\
0.001 \mathrm{a}\end{array}$ & $\begin{array}{r}0.111 \pm \\
0.003 b\end{array}$ & $\begin{array}{r}0.117 \pm \\
0.001 \mathrm{c}\end{array}$ & $\begin{array}{r}0.106 \pm \\
0.003 d\end{array}$ & $\begin{array}{r}0.098 \pm \\
0.001 \mathrm{~d}\end{array}$ & $\begin{array}{r}1.127 \pm \\
0.005 \mathrm{a}\end{array}$ & $\begin{array}{c}1.015 \pm \\
0.001 b\end{array}$ & $\begin{array}{r}1.003 \pm \\
0.005 \mathrm{c}\end{array}$ \\
\hline $\begin{array}{l}\text { Storage time }\left(14^{\text {th }}\right. \\
\text { day) }\end{array}$ & Control & $0.1 \%$ & $0.3 \%$ & $0.5 \%$ & Control & $0.1 \%$ & $0.3 \%$ & $0.5 \%$ & Control & $0.1 \%$ & $0.3 \%$ & $0.5 \%$ \\
\hline $\begin{array}{l}\text { Acidity } \\
(\mathrm{mgKOH} / 100 \mathrm{~g})\end{array}$ & $\begin{array}{r}1.63 \pm \\
0.13 \mathrm{a}\end{array}$ & $\begin{array}{r}1.47 \pm \\
0.09 \mathrm{~b}\end{array}$ & $\begin{array}{r}1.39 \pm \\
0.07 \mathrm{c}\end{array}$ & $\begin{array}{r}1.28 \pm \\
0.11 \mathrm{~d}\end{array}$ & $\begin{array}{r}1.74 \pm \\
0.15 \mathrm{a}\end{array}$ & $\begin{array}{r}1.71 \pm \\
0.09 \mathrm{~b}\end{array}$ & $\begin{array}{r}1.68 \pm \\
0.03 \mathrm{c}\end{array}$ & $\begin{array}{r}1.57 \pm \\
0.05 \mathrm{~d}\end{array}$ & $\begin{array}{r}1.83 \pm \\
0.17 \mathrm{~b}\end{array}$ & $\begin{array}{r}1.87 \pm \\
0.13 \mathrm{a}\end{array}$ & $\begin{array}{r}1.77 \pm \\
0.21 \mathrm{c}\end{array}$ & $\begin{array}{r}1.56 \pm \\
0.09 \mathrm{~d}\end{array}$ \\
\hline $\begin{array}{l}\text { Peroxide value } \\
\left(\mathrm{meqO}_{2} / \mathrm{kg}\right)\end{array}$ & $\begin{array}{c}12.76 \pm \\
0.45 \mathrm{a}\end{array}$ & $\begin{array}{r}9.63 \pm \\
0.36 \mathrm{~b}\end{array}$ & $\begin{array}{r}8.77 \pm \\
0.93 \mathrm{c}\end{array}$ & $\begin{array}{r}6.48 \pm \\
0.74 d\end{array}$ & $\begin{array}{c}15.67 \pm \\
0.65 \mathrm{a}\end{array}$ & $\begin{array}{c}13.86 \pm \\
0.58 b\end{array}$ & $\begin{array}{c}11.73 \pm \\
0.69 \mathrm{c}\end{array}$ & $\begin{array}{r}8.64 \pm \\
0.43 \mathrm{~d}\end{array}$ & $\begin{array}{c}18.46 \pm \\
0.52 \mathrm{a}\end{array}$ & $\begin{array}{c}16.35 \pm \\
0.38 b\end{array}$ & $\begin{array}{c}14.58 \pm \\
0.21 \mathrm{c}\end{array}$ & $\begin{array}{c}11.44 \pm \\
0.78 \mathrm{~d}\end{array}$ \\
\hline $\begin{array}{l}\text { Refractive Index } \\
\left(\mathrm{n}^{20} \mathrm{D}\right)\end{array}$ & $\begin{array}{r}1.531 \pm \\
0.003 \mathrm{a}\end{array}$ & $\begin{array}{l}1.527 \pm \\
0.001 \mathrm{bc}\end{array}$ & $\begin{array}{r}1.519 \pm \\
0.001 \mathrm{c}\end{array}$ & $\begin{array}{r}1.528 \pm \\
0.005 b\end{array}$ & $\begin{array}{r}1.510 \pm \\
0.001 \mathrm{~d}\end{array}$ & $\begin{array}{c}1.513 \pm \\
0.003 \mathrm{c}\end{array}$ & $\begin{array}{c}1.517 \pm \\
0.003 b\end{array}$ & $\begin{array}{c}1.521 \pm \\
0.005 \mathrm{a}\end{array}$ & $\begin{array}{c}1.488 \pm \\
0.001 \mathrm{~b}\end{array}$ & $\begin{array}{r}1.496 \pm \\
0.005 \mathrm{a}\end{array}$ & $\begin{array}{r}1.491 \pm \\
0.003 \mathrm{~d}\end{array}$ & $\begin{array}{r}1.485 \pm \\
0.001 \mathrm{c}\end{array}$ \\
\hline $\begin{array}{l}\text { Iodine value } \\
\left(\mathrm{gI}_{2} / 100 \mathrm{~g}\right)\end{array}$ & $\begin{array}{c}98.33 \pm \\
1.24 \mathrm{a}\end{array}$ & $\begin{array}{c}98.26 \pm \\
1.32 \mathrm{c}\end{array}$ & $\begin{array}{c}98.11 \pm \\
0.98 \mathrm{~d}\end{array}$ & $\begin{array}{c}98.29 \pm \\
0.76 \mathrm{~b}\end{array}$ & $\begin{array}{c}111.59 \pm \\
0.58 \mathrm{~b}\end{array}$ & $\begin{array}{c}111.57 \pm \\
1.16 \mathrm{c}\end{array}$ & $\begin{array}{c}111.49 \pm \\
1.27 \mathrm{~d}\end{array}$ & $\begin{array}{c}111.61 \pm \\
1.37 \mathrm{a}\end{array}$ & $\begin{array}{c}105.08 \pm \\
1.46 \mathrm{ab}\end{array}$ & $\begin{array}{c}105.01 \pm \\
1.32 \mathrm{c}\end{array}$ & $\begin{array}{c}104.97 \pm \\
1.48 \mathrm{~d}\end{array}$ & $\begin{array}{c}105.09 \pm \\
1.23 \mathrm{a}\end{array}$ \\
\hline $\begin{array}{l}\text { Saponification value } \\
(\mathrm{mgKOH} / 100 \mathrm{~g})\end{array}$ & $\begin{array}{c}192.94 \pm \\
1.45 \mathrm{a}\end{array}$ & $\begin{array}{c}192.81 \pm \\
1.38 \mathrm{~b}\end{array}$ & $\begin{array}{c}192.77 \pm \\
1.21 \mathrm{c}\end{array}$ & $\begin{array}{c}192.44 \pm \\
1.56 \mathrm{~d}\end{array}$ & $\begin{array}{c}194.32 \pm \\
1.73 \mathrm{a}\end{array}$ & $\begin{array}{c}194.15 \pm \\
1.49 \mathrm{c}\end{array}$ & $\begin{array}{c}194.01 \pm \\
2.56 \mathrm{~d}\end{array}$ & $\begin{array}{c}194.20 \pm \\
1.76 \mathrm{~b}\end{array}$ & $\begin{array}{c}193.17 \pm \\
1.38 \mathrm{a}\end{array}$ & $\begin{array}{c}193.05 \pm \\
1.71 \mathrm{~b}\end{array}$ & $\begin{array}{c}192.87 \pm \\
1.64 \mathrm{c}\end{array}$ & $\begin{array}{c}192.83 \pm \\
1.59 \mathrm{~d}\end{array}$ \\
\hline $\begin{array}{l}\text { Unsaponifiable matter } \\
(\%)\end{array}$ & $\begin{array}{r}1.69 \pm \\
0.13 \mathrm{a}\end{array}$ & $\begin{array}{r}1.60 \pm \\
0.21 b\end{array}$ & $\begin{array}{r}1.57 \pm \\
0.09 \mathrm{c}\end{array}$ & $\begin{array}{r}1.63 \pm \\
0.07 \mathrm{~d}\end{array}$ & $\begin{array}{r}1.84 \pm \\
0.05 \mathrm{a}\end{array}$ & $\begin{array}{r}1.80 \pm \\
0.11 \mathrm{~b}\end{array}$ & $\begin{array}{r}1.75 \pm \\
0.23 \mathrm{~b}\end{array}$ & $\begin{array}{r}1.78 \pm \\
0.27 \mathrm{c}\end{array}$ & $\begin{array}{r}1.16 \pm \\
0.09 \mathrm{a}\end{array}$ & $\begin{array}{r}1.14 \pm \\
0.05 \mathrm{c}\end{array}$ & $\begin{array}{r}1.12 \pm \\
0.09 \mathrm{~d}\end{array}$ & $\begin{array}{r}1.15 \pm \\
0.07 \mathrm{~b}\end{array}$ \\
\hline $\begin{array}{l}\text { Density } \\
\left(\mathrm{mg} / \mathrm{mL} ; 25^{\circ} \mathrm{C}\right)\end{array}$ & $\begin{array}{r}0.882 \pm \\
0.001 \mathrm{a}\end{array}$ & $\begin{array}{r}0.888 \pm \\
0.003 b\end{array}$ & $\begin{array}{r}0.895 \pm \\
0.001 \mathrm{c}\end{array}$ & $\begin{array}{l}0.897 \pm \\
0.002 \mathrm{~cd}\end{array}$ & $\begin{array}{r}0.852 \pm \\
0.001 \mathrm{~d}\end{array}$ & $\begin{array}{r}0.857 \pm \\
0.003 \mathrm{c}\end{array}$ & $\begin{array}{r}0.868 \pm \\
0.001 b\end{array}$ & $\begin{array}{r}0.877 \pm \\
0.003 \mathrm{a}\end{array}$ & $\begin{array}{r}0.873 \pm \\
0.001 \mathrm{~d}\end{array}$ & $\begin{array}{c}0.878 \pm \\
0.005 \mathrm{c}\end{array}$ & $\begin{array}{c}0.884 \pm \\
0.003 b\end{array}$ & $\begin{array}{c}0.893 \pm \\
0.001 \mathrm{a}\end{array}$ \\
\hline
\end{tabular}


K. Ghafoor, M. M. Özcan, F. A. Juhaimi et al.

Table 1 Continued.

\begin{tabular}{|c|c|c|c|c|c|c|c|c|c|c|c|c|}
\hline \multirow{2}{*}{$\begin{array}{c}\text { Storage time }\left(0^{\text {th }} \text { day }\right) \\
\text { Parameter }\end{array}$} & \multicolumn{4}{|c|}{ Orange (Barile) oil with sumac extract } & \multicolumn{4}{|c|}{ Lemon (Interdonato) oil with sumac extract } & \multicolumn{4}{|c|}{ Mandarin (Clementine) oil with sumac extract } \\
\hline & Control & $0.1 \%$ & $0.3 \%$ & $0.5 \%$ & Control & $0.1 \%$ & $0.3 \%$ & $0.5 \%$ & Control & $0.1 \%$ & $0.3 \%$ & $0.5 \%$ \\
\hline $\begin{array}{l}\text { Viscosity } \\
\left(\mathrm{Pa} . \mathrm{S} ; 25^{\circ} \mathrm{C}\right)\end{array}$ & $\begin{array}{r}0.138 \pm \\
0.013 \mathrm{~d}\end{array}$ & $\begin{array}{r}0.157 \pm \\
0.011 \mathrm{~b}\end{array}$ & $\begin{array}{r}0.169 \pm \\
0.021 \mathrm{a}\end{array}$ & $\begin{array}{r}0.145 \pm \\
0.033 \mathrm{c}\end{array}$ & $\begin{array}{r}0.174 \pm \\
0.017 \mathrm{c}\end{array}$ & $\begin{array}{r}0.187 \pm \\
0.015 \mathrm{a}\end{array}$ & $\begin{array}{r}0.184 \pm \\
0.013 b\end{array}$ & $\begin{array}{r}0.151 \pm \\
0.015 \mathrm{~d}\end{array}$ & $\begin{array}{r}0.150 \pm \\
0.009 \mathrm{~b}\end{array}$ & $\begin{array}{r}0.161 \pm \\
0.018 \mathrm{a}\end{array}$ & $\begin{array}{r}0.138 \pm \\
0.021 \mathrm{c}\end{array}$ & $\begin{array}{r}0.127 \pm \\
0.015 \mathrm{~d}\end{array}$ \\
\hline $\begin{array}{l}\text { Storage time }\left(21^{\text {st }}\right. \\
\text { day) }\end{array}$ & Control & $0.1 \%$ & $0.3 \%$ & $0.5 \%$ & Control & $0.1 \%$ & $0.3 \%$ & $0.5 \%$ & Control & $0.1 \%$ & $0.3 \%$ & $0.5 \%$ \\
\hline $\begin{array}{l}\text { Acidity } \\
\text { (mgKOH/100g) }\end{array}$ & $\begin{array}{r}1.87 \pm \\
0.03 \mathrm{a}\end{array}$ & $\begin{array}{l}1.65 \pm \\
0.13 b\end{array}$ & $\begin{array}{r}1.58 \pm \\
0.09 \mathrm{c}\end{array}$ & $\begin{array}{r}1.43 \pm \\
0.07 \mathrm{~d}\end{array}$ & $0.05 \mathrm{a}$ & $\begin{array}{r}1.87 \pm \\
0.03 \mathrm{~b}\end{array}$ & $\begin{array}{r}1.76 \pm \\
0.01 \mathrm{c}\end{array}$ & $\begin{array}{r}1.63 \pm \\
0.05 \mathrm{~d}\end{array}$ & $\begin{array}{r}1.99 \pm \\
0.11 \mathrm{~b}\end{array}$ & $\begin{array}{r}2.07 \pm \\
0.23 \mathrm{a}\end{array}$ & $\begin{array}{r}1.88 \pm \\
0.17 \mathrm{c}\end{array}$ & $\begin{array}{r}1.61 \pm \\
0.09 \mathrm{~d}\end{array}$ \\
\hline $\begin{array}{l}\text { Peroxide value } \\
\left(\mathrm{meqO}_{2} / \mathrm{kg}\right)\end{array}$ & $\begin{array}{r}24.76 \pm \\
1.34 \mathrm{a}\end{array}$ & $\begin{array}{c}13.98 \pm \\
1.21 \mathrm{~b}\end{array}$ & $\begin{array}{c}12.28 \pm \\
1.18 \mathrm{c}\end{array}$ & & $\begin{array}{c}28.44 \pm \\
1.32 \mathrm{a}\end{array}$ & $\begin{array}{c}17.28 \pm \\
1.18 \mathrm{~b}\end{array}$ & $\begin{array}{c}13.48 \pm \\
0.97 \mathrm{c}\end{array}$ & $\begin{array}{c}10.26 \pm \\
0.38 \mathrm{~d}\end{array}$ & $\begin{array}{c}31.67 \pm \\
1.56 \mathrm{a}\end{array}$ & $\begin{array}{c}25.86 \pm \\
1.58 \mathrm{~b}\end{array}$ & $\begin{array}{c}21.58 \pm \\
1.27 \mathrm{c}\end{array}$ & $\begin{array}{c}14.97 \pm \\
1.32 \mathrm{~d}\end{array}$ \\
\hline $\begin{array}{l}\text { Refractive Index } \\
\left(\mathrm{n}^{20} \mathrm{D}\right)\end{array}$ & & & & & & & & & & & & \\
\hline & $\begin{array}{c}98.30 \pm \\
0.56 \mathrm{a}\end{array}$ & & & & & $\begin{array}{c}111.55 \pm \\
0.77 \mathrm{c}\end{array}$ & & & & & & \\
\hline $\begin{array}{l}\text { Saponification value } \\
(\mathrm{mgKOH} / 100 \mathrm{~g})\end{array}$ & $\begin{array}{c}192.91 \pm \\
1.38 \mathrm{a}\end{array}$ & $\begin{array}{l}192.87 \pm \\
1.57 \mathrm{~b}\end{array}$ & $\begin{array}{c}192.75 \pm \\
1.62 \mathrm{c}\end{array}$ & & $\begin{array}{c}194.10 \pm \\
1.78 \mathrm{a}\end{array}$ & $\begin{array}{c}193.90 \pm \\
1.53 \mathrm{c}\end{array}$ & & & $\begin{array}{c}193.50 \pm \\
1.39 \mathrm{a}\end{array}$ & & & $\begin{array}{c}192.97 \pm \\
1.67 \mathrm{c}\end{array}$ \\
\hline $\begin{array}{l}\text { Unsaponifiable matter } \\
(\%)\end{array}$ & $\begin{array}{r}1.69 \pm \\
0.13 \mathrm{a}\end{array}$ & & & & & & & & & & & \\
\hline $\begin{array}{l}\text { Density } \\
\left(\mathrm{mg} / \mathrm{mL} ; 25^{\circ} \mathrm{C}\right)\end{array}$ & & & & & & & & & & & & \\
\hline $\begin{array}{l}\text { Viscosity } \\
\left(\mathrm{Pa} . \mathrm{S} ; 25^{\circ} \mathrm{C}\right)\end{array}$ & & & & & & $\begin{array}{c}0.293 \pm \\
0.15 b\end{array}$ & & & & & & $\begin{array}{c}0.178 \pm \\
0.15 \mathrm{~d}\end{array}$ \\
\hline $\begin{array}{l}\text { Storage time }\left(28^{\text {th }}\right. \\
\text { day) }\end{array}$ & Control & $0.1 \%$ & $0.3 \%$ & $0.5 \%$ & Control & $0.1 \%$ & $0.3 \%$ & $0.5 \%$ & Control & $0.1 \%$ & $0.3 \%$ & $0.5 \%$ \\
\hline $\begin{array}{l}\text { Acidity } \\
(\mathrm{mgKOH} / 100 \mathrm{~g})\end{array}$ & $\begin{array}{r}3.64 \pm \\
0.34 \mathrm{a}\end{array}$ & $\begin{array}{r}3.37 \pm \\
0.51 \mathrm{~b}\end{array}$ & $\begin{array}{r}3.13 \pm \\
0.29 \mathrm{c}\end{array}$ & & $\begin{array}{r}2.28 \pm \\
0.13 \mathrm{a}\end{array}$ & $\begin{array}{r}2.01 \pm \\
0.18 \mathrm{~b}\end{array}$ & $\begin{array}{r}1.88 \pm \\
0.27 \mathrm{c}\end{array}$ & $\begin{array}{r}1.67 \pm \\
0.15 \mathrm{~d}\end{array}$ & $\begin{array}{r}2.36 \pm \\
0.17 \mathrm{a}\end{array}$ & $\begin{array}{r}2.11 \pm \\
0.23 b\end{array}$ & $\begin{array}{r}1.88 \pm \\
0.21 \mathrm{c}\end{array}$ & $\begin{array}{r}1.74 \pm \\
0.18 \mathrm{~d}\end{array}$ \\
\hline $\begin{array}{l}\text { Peroxide value } \\
\left(\mathrm{meqO}_{2} / \mathrm{kg}\right)\end{array}$ & $\begin{array}{c}32.57 \pm \\
1.27 \mathrm{a}\end{array}$ & $\begin{array}{c}24.36 \pm \\
2.34 \mathrm{~b}\end{array}$ & $\begin{array}{c}18.44 \pm \\
1.18 \mathrm{c}\end{array}$ & $\begin{array}{r}11.68 \pm \\
1.67 \mathrm{~d}\end{array}$ & $\begin{array}{c}34.63 \pm \\
1.36 \mathrm{a}\end{array}$ & $\begin{array}{c}25.47 \pm \\
1.13 \mathrm{~b}\end{array}$ & $\begin{array}{c}18.78 \pm \\
1.28 \mathrm{c}\end{array}$ & $\begin{array}{r}12.55 \pm \\
1.134 \mathrm{~d}\end{array}$ & $\begin{array}{c}37.81 \pm \\
1.56 \mathrm{a}\end{array}$ & $\begin{array}{c}30.64 \pm \\
1.63 \mathrm{~b}\end{array}$ & $\begin{array}{l}23.87 \pm \\
1.28 \mathrm{c}\end{array}$ & $\begin{array}{c}17.56 \pm \\
1.17 \mathrm{~d}\end{array}$ \\
\hline $\begin{array}{l}\text { Refractive Index } \\
\left(\mathrm{n}^{20} \mathrm{D}\right)\end{array}$ & $\begin{array}{r}1.527 \pm \\
0.003 \mathrm{a}\end{array}$ & $\begin{array}{r}1.524 \pm \\
0.001 \mathrm{c}\end{array}$ & $\begin{array}{l}1.526 \pm \\
0.005 \mathrm{~b}\end{array}$ & $\begin{array}{r}1.526 \pm \\
0.001 b\end{array}$ & $\begin{array}{r}1.508 \pm \\
0.001 \mathrm{c}\end{array}$ & $\begin{array}{l}1.510 \pm \\
0.003 \mathrm{bc}\end{array}$ & $\begin{array}{r}1.511 \pm \\
0.003 b\end{array}$ & $\begin{array}{r}1.517 \pm \\
0.005 \mathrm{a}\end{array}$ & $\begin{array}{r}1.484 \pm \\
0.001 b\end{array}$ & $\begin{array}{r}1.480 \pm \\
0.003 \mathrm{~d}\end{array}$ & $\begin{array}{r}1.483 \pm \\
0.001 \mathrm{c}\end{array}$ & $\begin{array}{r}1.485 \pm \\
0.005 \mathrm{a}\end{array}$ \\
\hline $\begin{array}{l}\text { Iodine value } \\
\left(\mathrm{gI}_{2} / 100 \mathrm{~g}\right)\end{array}$ & $\begin{array}{c}98.27 \pm \\
1.32 \mathrm{a}\end{array}$ & $\begin{array}{c}98.25 \pm \\
1.21 \mathrm{~b}\end{array}$ & $\begin{array}{c}98.22 \pm \\
1.18 \mathrm{c}\end{array}$ & $\begin{array}{c}98.25 \pm \\
1.27 \mathrm{~b}\end{array}$ & $\begin{array}{c}111.55 \pm \\
1.46 \mathrm{a}\end{array}$ & $\begin{array}{l}111.51 \pm \\
1.53 \mathrm{~b}\end{array}$ & $\begin{array}{c}111.47 \pm \\
1.58 \mathrm{c}\end{array}$ & $\begin{array}{c}111.55 \pm \\
1.61 \mathrm{a}\end{array}$ & $\begin{array}{c}105.00 \pm \\
1.56 \mathrm{a}\end{array}$ & $\begin{array}{r}104.95 \pm \\
1.73 \mathrm{bc}\end{array}$ & $\begin{array}{c}104.91 \pm \\
1.49 \mathrm{c}\end{array}$ & $\begin{array}{c}104.98 \pm \\
1.38 \mathrm{~b}\end{array}$ \\
\hline $\begin{array}{l}\text { Saponification value } \\
(\mathrm{mgKOH} / 100 \mathrm{~g})\end{array}$ & $\begin{array}{c}192.90 \pm \\
1.25 \mathrm{a}\end{array}$ & $\begin{array}{c}192.88 \pm \\
1.18 \mathrm{~b}\end{array}$ & $\begin{array}{c}192.84 \pm \\
1.61 \mathrm{c}\end{array}$ & $\begin{array}{c}192.68 \pm \\
1.45 \mathrm{~d}\end{array}$ & $\begin{array}{c}194.07 \pm \\
1.32 \mathrm{a}\end{array}$ & $\begin{array}{l}193.91 \pm \\
1.57 \mathrm{~b}\end{array}$ & $\begin{array}{c}193.86 \pm \\
1.49 \mathrm{c}\end{array}$ & $\begin{array}{c}194.05 \pm \\
1.35 \mathrm{ab}\end{array}$ & $\begin{array}{c}193.45 \pm \\
1.37 \mathrm{a}\end{array}$ & $\begin{array}{c}193.41 \pm \\
1.65 \mathrm{~b}\end{array}$ & $\begin{array}{c}193.32 \pm \\
1.53 \mathrm{c}\end{array}$ & $\begin{array}{c}193.21 \pm \\
1.38 \mathrm{~d}\end{array}$ \\
\hline $\begin{array}{l}\text { Unsaponifiable matter } \\
\text { (\%) }\end{array}$ & $\begin{array}{r}1.68 \pm \\
0.13 \mathrm{a}\end{array}$ & $\begin{array}{r}1.64 \pm \\
0.17 \mathrm{~b}\end{array}$ & $\begin{array}{r}1.62 \pm \\
0.09 \mathrm{c}\end{array}$ & $\begin{array}{r}1.58 \pm \\
0.05 \mathrm{~d}\end{array}$ & $\begin{array}{r}1.80 \pm \\
0.07 \mathrm{a}\end{array}$ & $\begin{array}{r}1.77 \pm \\
0.18 \mathrm{~b}\end{array}$ & $\begin{array}{l}1.75 \pm \\
0.11 \mathrm{bc}\end{array}$ & $\begin{array}{l}1.79 \pm \\
0.21 \mathrm{ab}\end{array}$ & $\begin{array}{r}1.14 \pm \\
0.09 \mathrm{a}\end{array}$ & $\begin{array}{l}1.11 \pm \\
0.05 \mathrm{bc}\end{array}$ & $\begin{array}{r}1.07 \pm \\
0.03 \mathrm{c}\end{array}$ & $\begin{array}{l}1.12 \pm \\
0.05 \mathrm{~b}\end{array}$ \\
\hline $\begin{array}{l}\text { Density } \\
\left(\mathrm{mg} / \mathrm{mL} ; 25^{\circ} \mathrm{C}\right)\end{array}$ & $\begin{array}{r}0.880 \pm \\
0.001 \mathrm{~d}\end{array}$ & $\begin{array}{r}0.887 \pm \\
0.003 \mathrm{c}\end{array}$ & $\begin{array}{r}0.892 \pm \\
0.005 b\end{array}$ & $\begin{array}{r}0.899 \pm \\
0.007 \mathrm{a}\end{array}$ & $\begin{array}{r}0.851 \pm \\
0.003 \mathrm{~d}\end{array}$ & $\begin{array}{r}0.859 \pm \\
0.005 \mathrm{c}\end{array}$ & $\begin{array}{r}0.864 \pm \\
0.005 b\end{array}$ & $\begin{array}{r}0.876 \pm \\
0.001 \mathrm{a}\end{array}$ & $\begin{array}{r}0.870 \pm \\
0.009 \mathrm{~d}\end{array}$ & $\begin{array}{r}0.874 \pm \\
0.005 \mathrm{c}\end{array}$ & $\begin{array}{r}0.875 \pm \\
0.005 b\end{array}$ & $\begin{array}{r}0.879 \pm \\
0.001 \mathrm{a}\end{array}$ \\
\hline $\begin{array}{l}\text { Viscosity } \\
\left(\mathrm{Pa} . \mathrm{S} ; 25^{\circ} \mathrm{C}\right)\end{array}$ & $\begin{array}{r}0.675 \pm \\
0.013 \mathrm{a}\end{array}$ & $\begin{array}{r}0.476 \pm \\
0.015 \mathrm{~b}\end{array}$ & $\begin{array}{r}0.389 \pm \\
0.021 \mathrm{c}\end{array}$ & $\begin{array}{r}0.123 \pm \\
0.017 \mathrm{~d}\end{array}$ & $\begin{array}{r}0.697 \pm \\
0.009 \mathrm{a}\end{array}$ & $\begin{array}{r}0.613 \pm \\
0.007 \mathrm{~b}\end{array}$ & $\begin{array}{r}0.583 \pm \\
0.023 \mathrm{c}\end{array}$ & $\begin{array}{r}0.257 \pm \\
0.018 \mathrm{~d}\end{array}$ & $\begin{array}{r}0.728 \pm \\
0.029 \mathrm{a}\end{array}$ & $\begin{array}{r}0.547 \pm \\
0.031 \mathrm{~b}\end{array}$ & $\begin{array}{r}0.326 \pm \\
0.019 \mathrm{c}\end{array}$ & $\begin{array}{r}0.215 \pm \\
0.009 \mathrm{~d}\end{array}$ \\
\hline
\end{tabular}

$*$ mean \pm standard deviation; **Values within each row followed by different letters are significantly different $(p<0.05)$

and $1.63 \mathrm{mgKOH} / 100 \mathrm{~g}(0.1 \%$ extract) for mandarin oil. Peroxide values at the $7^{\text {th }}$ day of storage changed between $2.63 \mathrm{meqO}_{2} / \mathrm{kg}\left(0.5 \%\right.$ extract) and $5.27 \mathrm{meqO}_{2} / \mathrm{kg}$ (control) for orange oil, $4.61 \mathrm{meqO}_{2} / \mathrm{kg}(0.5 \%$ extract $)$ and 6.35 $\mathrm{meqO}_{2} / \mathrm{kg}$ (control) for lemon oil and $5.07 \mathrm{meqO}_{2} / \mathrm{kg}(0.5 \%$ extract) and $7.98 \mathrm{meqO}_{2} / \mathrm{kg}$ (control) in case of mandarin oil. Partial significant differences were observed in case of refractive index and iodine values of treated oils depending on extract concentrations. The density values were between $0.883 \mathrm{mg} / \mathrm{mL}$ (control) and $0.899 \mathrm{mg} / \mathrm{mL}(0.5 \%$ extract) in case of orange oil, $0.855 \mathrm{mg} / \mathrm{mL}$ (control) and $0.875 \mathrm{mg} / \mathrm{mL}$ ( $0.5 \%$ extract) in lemon oil and $0.875 \mathrm{mg} / \mathrm{mL}$ (control) and $0.892 \mathrm{mg} / \mathrm{mL}$ ( $0.5 \%$ extract) for mandarin oil. The viscosity values were found between $0.093 \mathrm{~Pa} . \mathrm{S}$ (control) and 0.109 Pa.S (0.3\% extract) in orange oil, 0.105 Pa.S (control) and 0.117 Pa.S (0.3\% extract) in lemon oil and $0.098 \mathrm{~Pa} . \mathrm{S}$ (control) and $1.127 \mathrm{Pa.S}(0.1 \%$ extract) in mandarin oil

After $14^{\text {th }}$ day of storage, the acidity values varied between $1.28 \mathrm{mgKOH} / 100 \mathrm{~g}(0.5 \%$ extract $)$ and 1.63 $\mathrm{mgKOH} / 100 \mathrm{~g}$ (control) in orange oil, $1.57 \mathrm{mgKOH} / 100 \mathrm{~g}$ $(0.5 \%$ extract) and $1.74 \mathrm{mgKOH} / 100 \mathrm{~g}$ (control) in lemon oil and $1.56 \mathrm{mgKOH} / 100 \mathrm{~g}(0.5 \%$ extract $)$ and 1.83 $\mathrm{mgKOH} / 100 \mathrm{~g}$ (control) in case of mandarin oil. The perox- 


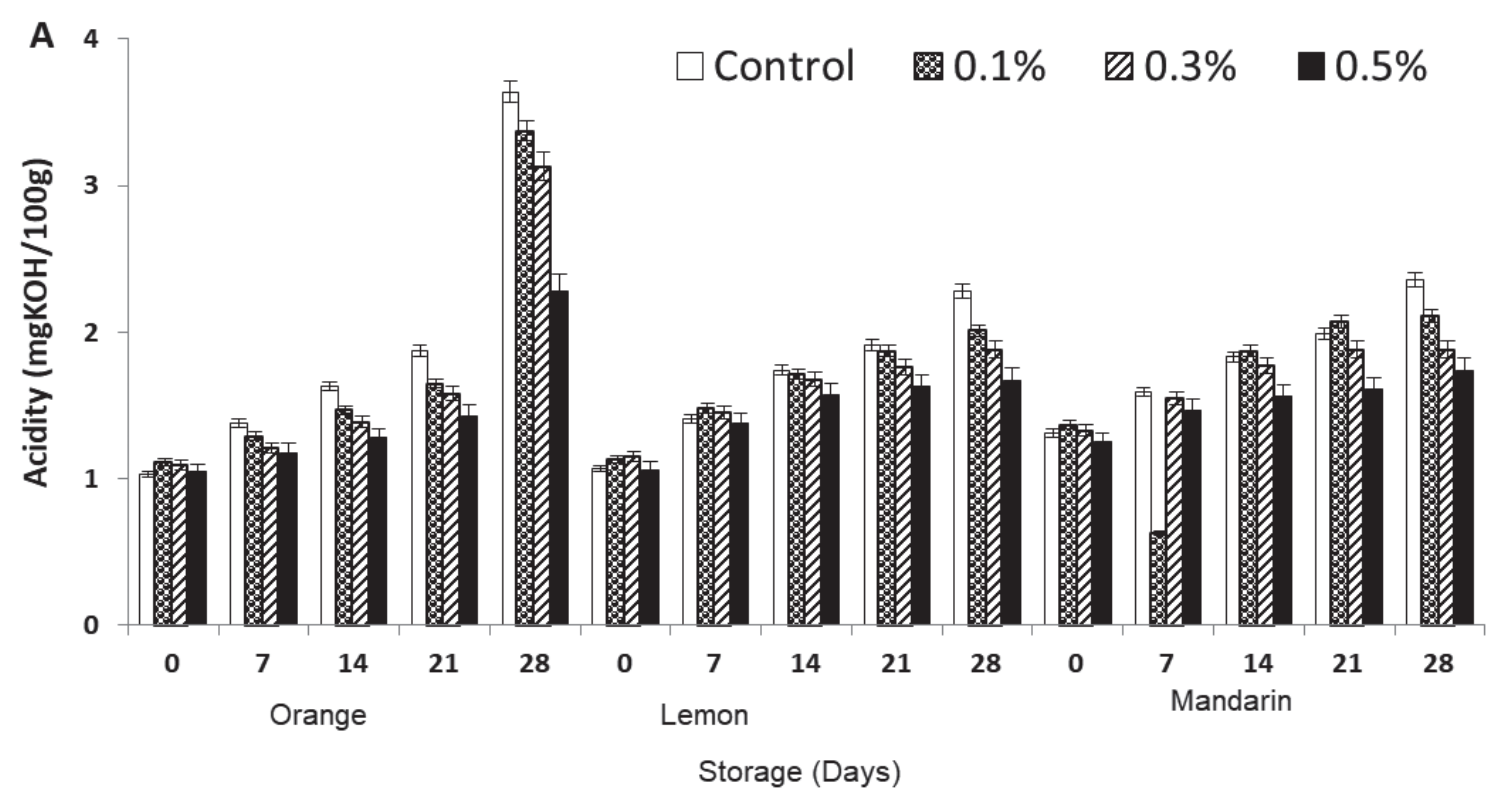

Fig. 1 Acidity values of citrus oils treated by sumac extract at different concentrations.

ide values changed between $6.48 \mathrm{meqO}_{2} / \mathrm{kg}(0.5 \%$ extract $)$ and $12.76 \mathrm{meqO}_{2} / \mathrm{kg}$ (control) in orange oil, $8.64 \mathrm{meqO}_{2} / \mathrm{kg}$ $\left(0.5 \%\right.$ extract) and $15.67 \mathrm{meqO}_{2} / \mathrm{kg}$ (control) lemon oil and $11.44 \mathrm{meqO}_{2} / \mathrm{kg}(0.5 \%$ extract $)$ and $18.46 \mathrm{meqO}_{2} / \mathrm{kg}$ (control) in mandarin oil after $14^{\text {th }}$ day. Partially significant differences were observed among refractive index, iodine and saponification values of sumac treated citrus oils at the $14^{\text {th }}$ day of storage. The saponfiable values of treated oil samples partially decreased with increasing extract concentration. The density values varied between $0.882 \mathrm{mg} /$ $\mathrm{mL}$ (control) and $0.897 \mathrm{mg} / \mathrm{mL}(0.5 \%$ extract) in orange oil, between $0.852 \mathrm{mg} / \mathrm{mL}$ (control) and $0.877 \mathrm{mg} / \mathrm{mL}(0.5 \%$ extract) in lemon oil and between $0.873 \mathrm{mg} / \mathrm{mL}$ (control) and $0.893 \mathrm{mg} / \mathrm{mL}(0.5 \%$ extract $)$ in mandarin oil after treatment with sumac extract. The viscosity values of samples varied between 0.138 Pa.S (control) and 0.169 Pa.S (0.3\% extract) in orange oil, $0.151 \mathrm{Pa.S}(0.5 \%$ extract) and 0.187 Pa.S (0.1\% extract) in lemon oil and $0.127 \mathrm{Pa.S}(0.5 \%$ extract) and $0.161 \mathrm{Pa.S}(0.1 \%$ extract $)$ in mandarin oil samples.

The analytical results on $21^{\text {st }}$ day of storage showed that acidity values remained $1.43 \mathrm{mgKOH} / 100 \mathrm{~g}(0.5 \%$ extract) to $1.87 \mathrm{mgKOH} / 100 \mathrm{~g}$ (control)in orange oil, 1.63 $\mathrm{mgKOH} / 100 \mathrm{~g}(0.5 \%$ extract) to $1.91 \mathrm{mgKOH} / 100 \mathrm{~g}$ (control) in lemon oil and $1.61 \mathrm{mgKOH} / 100 \mathrm{~g}(0.5 \%$ extract $)$ to 2.07 mgKOH/100g $(0.1 \%$ extract $)$ in mandarin oil samples. The peroxide values were found between $8.57 \mathrm{meqO}_{2} / \mathrm{kg}(0.5 \%$ extract) and $24.76 \mathrm{meqO}_{2} / \mathrm{kg}$ (control) in orange oil, 10.26 $\mathrm{meqO}_{2} / \mathrm{kg}\left(0.5 \%\right.$ extract) and $28.44 \mathrm{meqO}_{2} / \mathrm{kg}$ (control) in lemon oil and $14.97 \mathrm{meqO}_{2} / \mathrm{kg}(0.5 \%$ extract $)$ and 31.67 $\mathrm{meqO}_{2} / \mathrm{kg}$ (control) in mandarin oil samples. The unsaponifiable matter values were determined between $1.59 \%$ ( $0.5 \%$ extract) and $1.69 \%$ (control) in orange oil and $1.77 \%$
( $0.3 \%$ extract) and $1.81 \%$ (control) in lemon oil. The density values were $0.881 \mathrm{mg} / \mathrm{mL}$ (control) to $0.901 \mathrm{mg} / \mathrm{mL}$ ( $0.5 \%$ extract) in orange oil, $0.853 \mathrm{mg} / \mathrm{mL}$ (control) to 0.879 $\mathrm{mg} / \mathrm{mL}(0.5 \%$ extract $)$ in lemon oil and $0.872 \mathrm{mg} / \mathrm{mL}$ (control) to $0.880 \mathrm{mg} / \mathrm{mL}(0.5 \%$ extract $)$ in mandarin oil. The viscosity values varied between 0.187 Pa.S (0.3\% extract) and $0.257 \mathrm{~Pa} . \mathrm{S}$ (control) in orange oil, 0.178 Pa.S ( $0.5 \%$ extract) to $0.311 \mathrm{~Pa} . \mathrm{S}$ (control) in lemon oil.

At the $28^{\text {th }}$ day of storage, acidity values were detected between $2.28 \mathrm{mgKOH} / 100 \mathrm{~g}(0.5 \%$ extract $)$ and 3.64 $\mathrm{mgKOH} / 100 \mathrm{~g}$ (control) in orange oil, $1.67 \mathrm{mgKOH} / 100 \mathrm{~g}$ $(0.5 \%$ extract) and $2.28 \mathrm{mgKOH} / 100 \mathrm{~g}$ (control) in mandarin oil and $1.74 \mathrm{mgKOH} / 100 \mathrm{~g}(0.5 \%$ extract $)$ and 2.36 $\mathrm{mgKOH} / 100 \mathrm{~g}$ (control) in mandarin oil samples after addition of sumac extracts (Fig. 1). The peroxide values were found between $11.68 \mathrm{meqO}_{2} / \mathrm{kg}(0.5 \%$ extract $)$ and 32.57 $\mathrm{meqO}_{2} / \mathrm{kg}$ (control) in orange oil, $12.55 \mathrm{meqO}_{2} / \mathrm{kg}(0.5 \%$ extract) and $34.63 \mathrm{meqO}_{2} / \mathrm{kg}$ (control) in lemon oil and $17.56 \mathrm{meqO}_{2} / \mathrm{kg}(0.5 \%$ extract $)$ and $37.81 \mathrm{meqO}_{2} / \mathrm{kg}$ (control) in mandarin oil samples (Fig. 2). The unsaponifiable matter values of oil samples changed between 1.58\% ( $0.5 \%$ extract) and $1.68 \%$ (control) for orange, $1.75 \%$ ( $0.3 \%$ extract) and $1.80 \%$ (control) for lemon, and $1.07 \%$ ( $0.3 \%$ extract) and $1.14 \%$ (control) for mandarin oil samples. The density values of citrus seed oils treated with sumac extract at different concentrations also showed changes, and varied between $0.880 \mathrm{mg} / \mathrm{mL}$ (control) and $0.899 \mathrm{mg} / \mathrm{mL}(0.5 \%$ extract) for orange, $0.851 \mathrm{mg} / \mathrm{mL}$ (control) and $0.876 \mathrm{mg} / \mathrm{mL}(0.5 \%$ extract) for lemon and $0.870 \mathrm{mg} / \mathrm{mL}$ (control) and $0.879 \mathrm{mg} / \mathrm{mL}(0.5 \%$ extract) for mandarin seed oil samples (Fig. 3). The viscosity values of samples changed between $0.123 \mathrm{~Pa} . \mathrm{S}(0.5 \%$ extract $)$ and $0.675 \mathrm{~Pa} . \mathrm{S}$ (control) in orange, between $0.257 \mathrm{~Pa} . \mathrm{S}(0.5 \%$ 


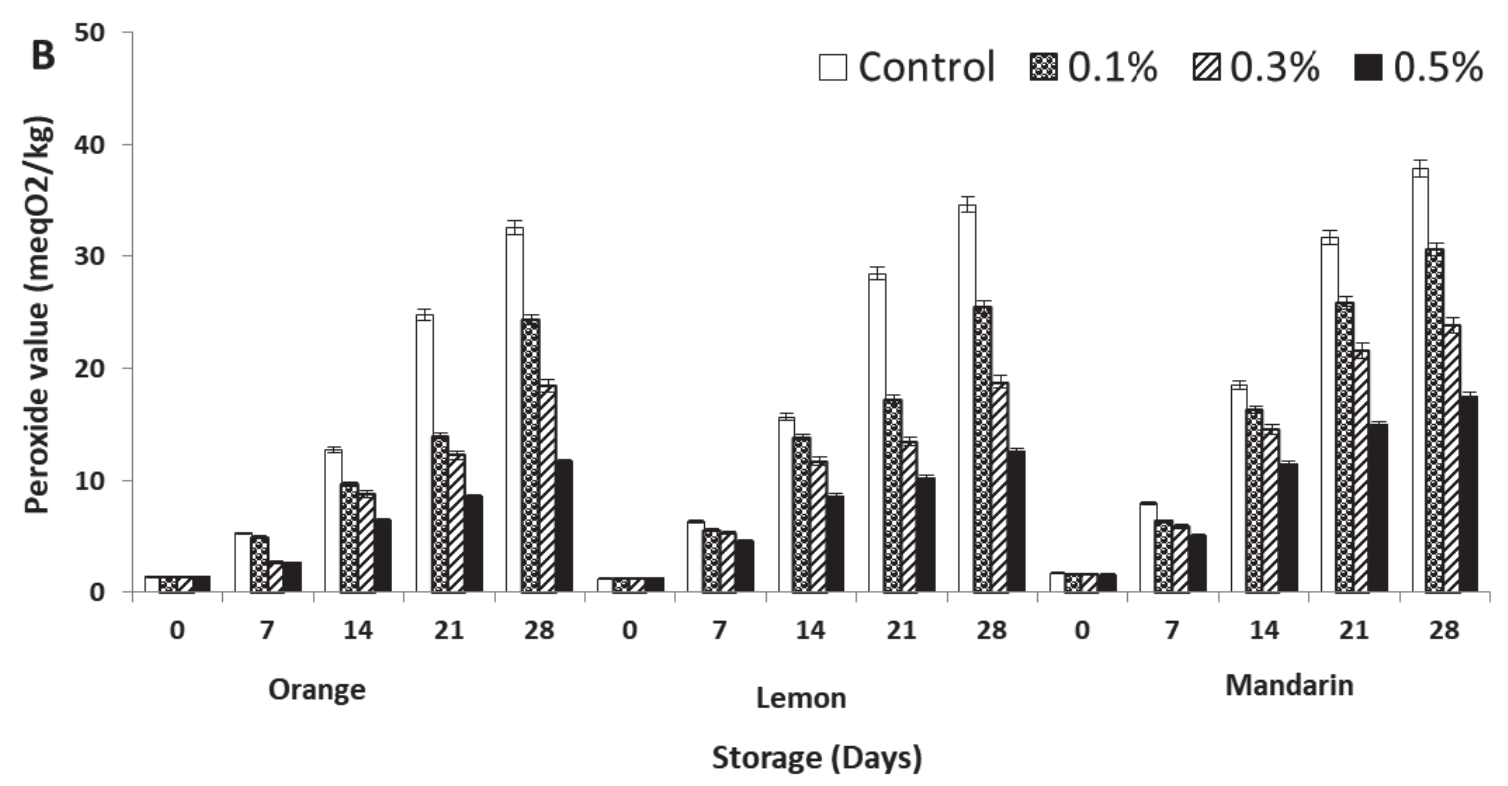

Fig. 2 Peroxide values of citrus oils treated by sumac extract at different concentrations.

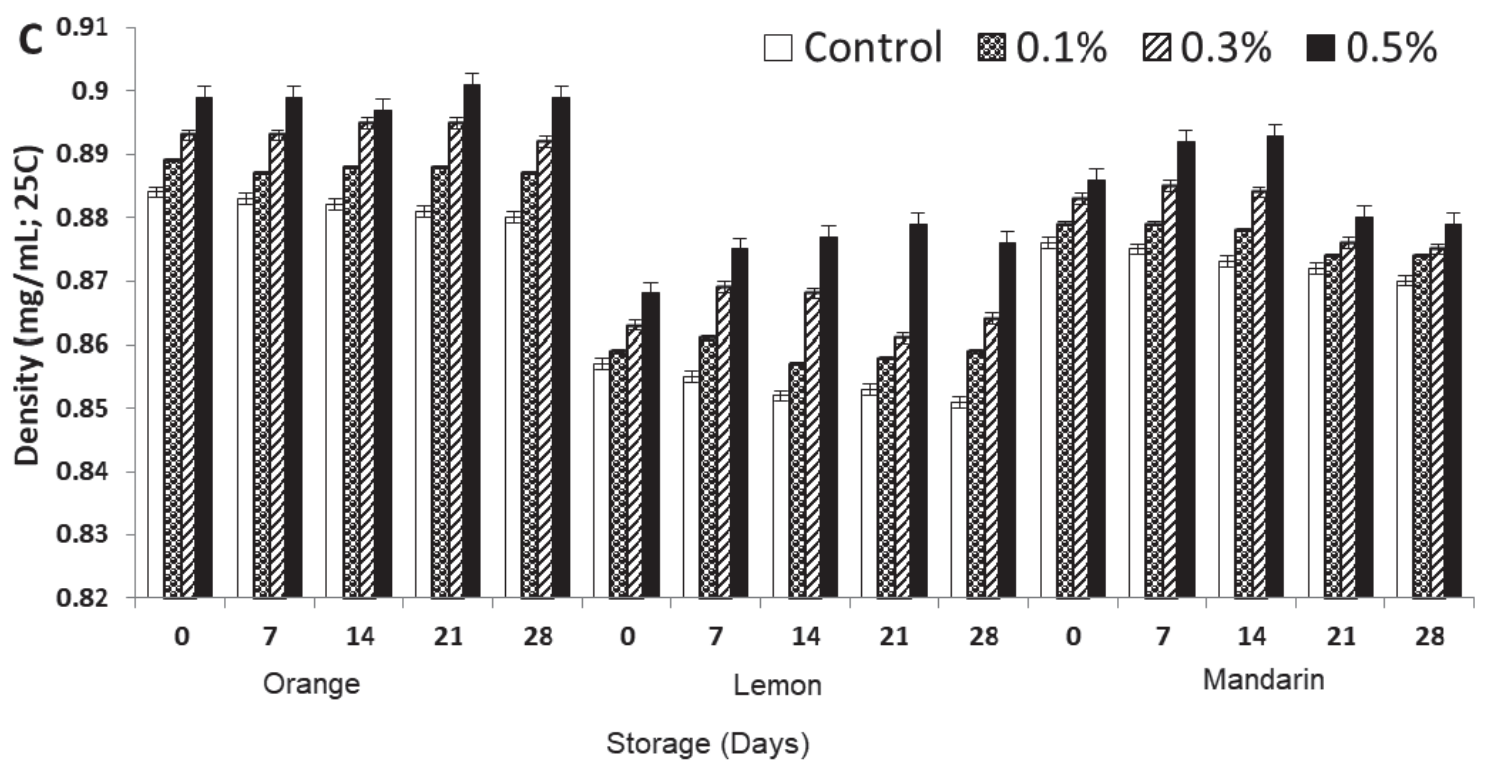

Fig. 3 Density values of citrus oils treated by sumac extract at different concentrations.

extract) and $0.697 \mathrm{~Pa} . \mathrm{S}$ (control) in lemon and from 0.215 Pa.S (0.5\% extract) and 0.728 Pa.S (control) in mandarin oil samples (Fig. 4).

Depending on storage periods of citrus seed oils, the acidity values changed between $1.03 \mathrm{mgKOH} / 100 \mathrm{~g}\left(0^{\text {th }}\right.$ day $)$ and $3.64 \mathrm{mgKOH} / 100 \mathrm{~g}\left(28^{\text {th }}\right.$ day) for orange oil samples. The acidity values of orange oil containing $0.5 \%$ sumac extract were determined to be $1.05 \mathrm{mgKOH} / 100 \mathrm{~g}\left(0^{\text {th }}\right.$ day $)$ and $2.28 \mathrm{mgKOH} / 100 \mathrm{~g}\left(28^{\text {th }}\right.$ day $)$. The acidity values of lemon oils for control group varied between 1.07 $\mathrm{mgKOH} / 100 \mathrm{~g}\left(0^{\text {th }}\right.$ day $)$ and $2.28 \mathrm{mgKOH} / 100 \mathrm{~g}\left(28^{\text {th }}\right.$ day $)$ while that of samples treated with $0.5 \%$ sumac extract changed between $1.06 \mathrm{mgKOH} / 100 \mathrm{~g}\left(0^{\text {th }}\right.$ day) and 1.67
mgKOH/100g ( $28^{\text {th }}$ day). The acidity values of mandarin oils for control group changed between $1.31 \mathrm{mgKOH} / 100 \mathrm{~g}\left(0^{\text {th }}\right.$ day) and $2.36 \mathrm{mgKOH} / 100 \mathrm{~g}\left(28^{\text {th }}\right.$ day $)$ whereas in case of oil sample with $0.5 \%$ sumac extract concentration, acidity value varied between $1.25 \mathrm{mgKOH} / 100 \mathrm{~g}\left(0^{\text {th }}\right.$ day $)$ and 1.74 $\mathrm{mgKOH} / 100 \mathrm{~g}\left(28^{\text {th }}\right.$ day $)$.

During storage of citrus oils treated with sumac extracts at varying concentrations, peroxide values were observed in the ranges of $1.38\left(0^{\text {th }}\right.$ day $)$ and $32.57 \mathrm{meqO}_{2} / \mathrm{kg}\left(28^{\text {th }}\right.$ day $)$ in orange control, $1.24 \mathrm{meqO}_{2} / \mathrm{kg}\left(0^{\text {th }}\right.$ day $)$ and $34.63 \mathrm{meqO}_{2} /$ $\mathrm{kg}\left(28^{\text {th }}\right.$ day $)$ in lemon control group and $1.71 \mathrm{meqO}_{2} / \mathrm{kg}\left(0^{\text {th }}\right.$ day) and $37.81 \mathrm{meqO}_{2} / \mathrm{kg}\left(28^{\text {th }}\right.$ day) in mandarin control samples. The peroxide values of citrus oils with $0.5 \%$ 


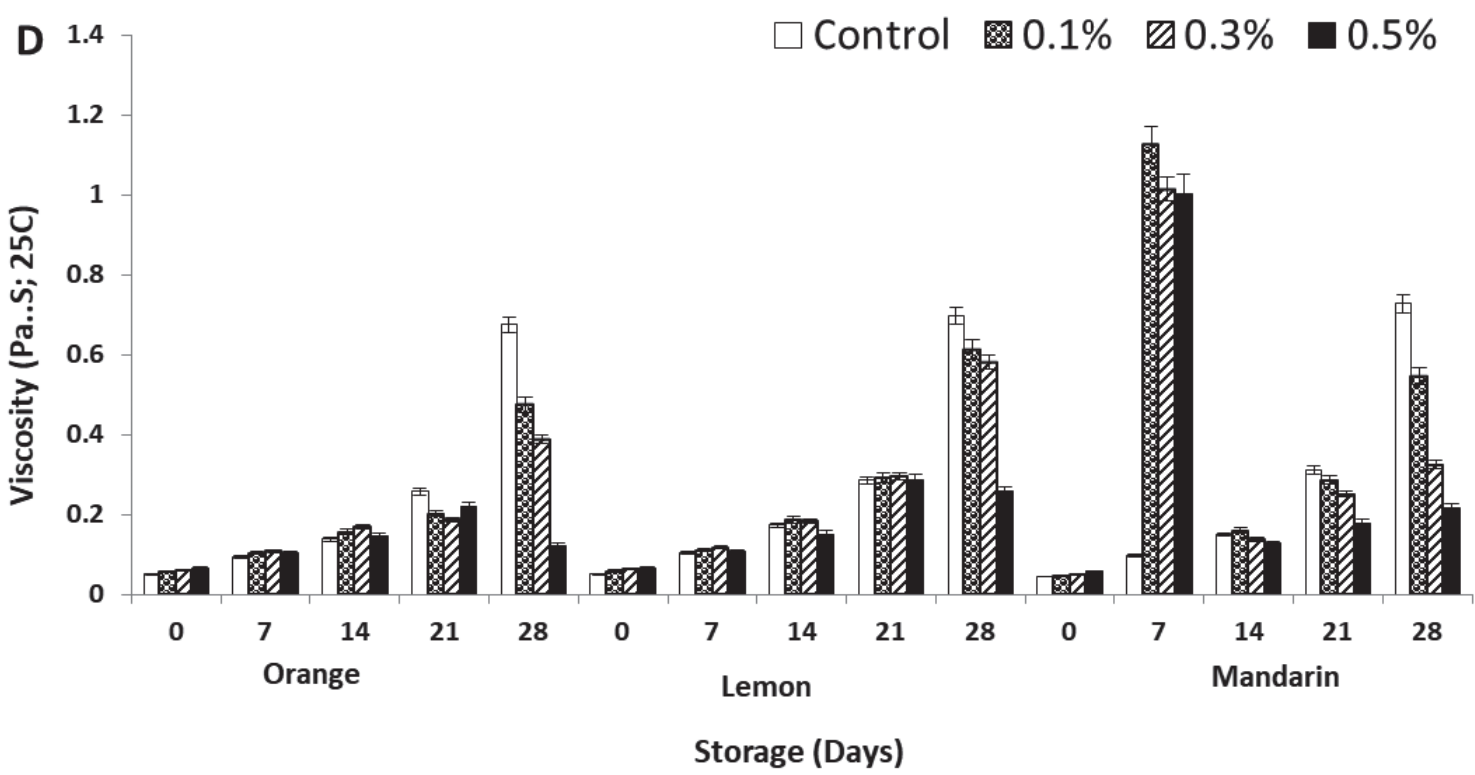

Fig. 4 Viscosity values of citrus oils treated by sumac extract at different concentrations.

sumac extract were determined between $1.37 \mathrm{meqO}_{2} / \mathrm{kg}\left(0^{\text {th }}\right.$ day) and $12.55 \mathrm{meqO}_{2} / \mathrm{kg}\left(28^{\text {th }}\right.$ day) for orange, $1.26 \mathrm{meqO}_{2} /$ $\mathrm{kg}\left(0^{\text {th }}\right.$ day $)$ and $12.55 \mathrm{meqO}_{2} / \mathrm{kg}\left(28^{\text {th }}\right.$ day $)$ for lemon and $1.60 \mathrm{meqO}_{2} / \mathrm{kg}\left(0^{\text {th }}\right.$ day $)$ and $17.56 \mathrm{meqO}_{2} / \mathrm{kg}\left(28^{\text {th }}\right.$ day $)$ for mandarin seed oils. The refractive index values changed between 1.541 ( $0^{\text {th }}$ day $)$ and $1.527\left(28^{\text {th }}\right.$ day $)$ for orange control while that of lemon oils with $0.5 \%$ sumac extract varied between $1.514\left(0^{\text {th }}\right.$ day $)$ and $1.517\left(28^{\text {th }}\right.$ day $)$ and in case of mandrain control, 1.491 ( $0^{\text {th }}$ day $)$ and $1.484\left(28^{\text {th }}\right.$ day). The refractive index values of mandarin oil with $0.5 \%$ extract varied between $1.478\left(0^{\text {th }}\right.$ day $)$ and $1.485\left(28^{\text {th }}\right.$ day). Depending on storage periods, the density values of control orange oils were measured between $0.884 \mathrm{mg} / \mathrm{mL}$ $\left(0^{\text {th }}\right.$ day $)$ and $0.880 \mathrm{mg} / \mathrm{mL}\left(28^{\text {th }}\right.$ day $)$ while the density values of orange oils with $0.5 \%$ sumac extract were between $0.899 \mathrm{mg} / \mathrm{mL}\left(0^{\text {th }}\right.$ day $)$ and $0.899 \mathrm{mg} / \mathrm{mL}\left(28^{\text {th }}\right.$ day $)$. The density values were between $0.857 \mathrm{mg} / \mathrm{mL}\left(0^{\text {th }}\right.$ day $)$ and $0.851 \mathrm{mg} / \mathrm{mL}$ ( $28^{\text {th }}$ day) control lemon oil whereas that of lemon oil with $0.5 \%$ sumac extract varied between 0.868 $\mathrm{mg} / \mathrm{mL}\left(0^{\text {th }}\right.$ day $)$ and $0.876 \mathrm{mg} / \mathrm{mL}$ ( $28^{\text {th }}$ day $)$. In addition, density values of control mandarin oil changed between $0.876 \mathrm{mg} / \mathrm{mL}$ ( $0^{\text {th }}$ day $)$ and $0.870 \mathrm{mg} / \mathrm{mL}$ ( $28^{\text {th }}$ day $)$ while that of mandarin oil with $0.5 \%$ sumac extract were determined between $0.886 \mathrm{mg} / \mathrm{mL}$ ( $0^{\text {th }}$ day $)$ and $0.879 \mathrm{mg} / \mathrm{mL}\left(28^{\text {th }}\right.$ day $)$. The viscosity values of control orange oil samples depending on storage times were measured between $0.051 \mathrm{~Pa} . \mathrm{S}$ $\left(0^{\text {th }}\right.$ day $)$ and 0.675 Pa.S $\left(28^{\text {th }}\right.$ day) while that of orange oils with $0.5 \%$ sumac extract were detected between 0.065 $\mathrm{Pa} . \mathrm{S}$ ( $0^{\text {th }}$ day $)$ and $0.123 \mathrm{~Pa} . \mathrm{S}\left(28^{\text {th }}\right.$ day). In addition, the viscosity values of control lemon oil changed between 0.051 Pa.S ( ${ }^{\text {th }}$ day) and 0.698 Pa.S ( $28^{\text {th }}$ day) whereas samples containing $0.5 \%$ sumac extract showed a viscosity variation from $0.067 \mathrm{~Pa} . \mathrm{S}\left(0^{\text {th }}\right.$ day) to $0.257 \mathrm{~Pa} . \mathrm{S}\left(28^{\text {th }}\right.$ day). The viscosity values of control mandarin oil changed between $0.044 \mathrm{~Pa} . \mathrm{S}\left(0^{\text {th }}\right.$ day $)$ and $0.728 \mathrm{~Pa} . \mathrm{S}\left(28^{\text {th }}\right.$ day $)$ while that of mandarin oil with $0.5 \%$ sumac extract were between 0.057 $\operatorname{Pa.S}\left(0^{\text {th }}\right.$ day) and $0.215 \mathrm{~Pa} . \mathrm{S}\left(28^{\text {th }}\right.$ day $)$. Hence, the acidity, peroxide and viscosity values of control citrus oils increased with storage time whereas acidity, peroxide, refractive index, unsaponifiable matter and density values of citrus oils treated with sumac extract at different concentrations decreased with increasing storage time. The most effective extract concentration was found to be $0.5 \%$ in terms of these parameters. Other concentrations showed partial effects on physico-chemical properties and storage stability of citrus oils. Differences were noted on physicochemical properties of citrus oils with sumac extracts added at different concentrations and with changes storage periods. These differences can be probably due to physicochemical properties of both sumac extracts and citrus oils at different storage times. In addition, these differences might also resulted due to the biochemical reactions of citrus oil and sumac extracts. In general, the partial rise in the viscosity of citrus oils may be due to partial polymerization of the oil in the presence of oxygen. Almost all the concentrations of sumac extracts affected peroxide values and acidity values of citrus oils. Generally, the highest effect on acidity and peroxide values were observed by the $0.5 \%$ concentrations of sumac extract. Sumac, which is grown in Mediterranean and Southeastern Anatolian regions of Turkey, was utilized by Özcan and Akgül ${ }^{11,17)}$ for sunflower oil stability and observed beneficial effects. Bashash et al. ${ }^{18)}$ reported that water extracts of sumac exhibited effective antioxidant and radical scavenging activities as compared extracts prepared in other solvents. The antioxidant capacity values were between 58.802\% (Brown 
sumac powder) and $82.566 \%$ (Brown sumac fruit) for sumac extract ethanol, between $81.243 \%$ (Brown sumac powder) and $86.709 \%$ (red sumac powder) for sumac distilled water extract ${ }^{8)}$. Mazaheri Tehrani et $a l .{ }^{18)}$ reported that the antioxidant activity, free radical scavenging and reducing capacity of sumac extract in ethanol were higher than those of the aqueous one. It has been observed that the three types of sumac seed extracts (SSEs) exhibited different antioxidant activities in another study ${ }^{19)}$ wherein the methanolic SSEs contained larger amounts of phenolic and flavonoid compounds and have higher antioxidant activities than those of aqueous or ethanolic SSEs. Staghorn sumac (Rhus hirta L.) is a native tree in Eastern Canada whose fruit has been used by aboriginal peoples to treat various illnesses, and has recently been found to be a good source of antioxidant ${ }^{20)}$. Oxidative stability of black cumin, coriander and niger crude and stripped seed oils was determined and compared. According to results, oxidative stabilities of crude oils were stronger than their stripped counterparts and the order of oxidative stability was as follows: coriander $>$ black cumin $>$ niger seed $^{21)}$. The antioxidant properties of potato peels and sugar beet pulp, in comparison with synthetic antioxidant, were investigated. The order of oxidative stability was as follow: TBHQ $>$ potato peels $>$ BHT $=$ sugar beet pulp $>$ BHA $^{22)}$. Kıralan and Ramadan ${ }^{23}$ investigated the effect of heating and microwave treatment on the levels of volatile oxidation products and the stability of safflower (Carthamus tinctorius L.), sesame (Sesamum indicum) and canola (Brassica napus L.) cold-pressed oils. 2-Heptenal and 2,4-heptadienal isomers were determined as key constituents in canola oil, while hexanal and 2-heptenal are found in high levels in safflower and sesame oils in oven conditions ${ }^{23}$. Current study also reports an effective utilization of sumac extracts, prepared in methanol, for stabilizing certain physico-chemical properties of citrus oil stored for 28 days at higher than ambient temperatures. Further studies can be carried out on evaluating functional and biological properties of citrus seed oil containing sumac fruit extract.

\section{Conclusion}

It can be inferred that after addition of sumac flower extract to oil samples from seed of orange, lemon and mandarin different physico-chemical properties were affected. For instance, the peroxide and acidity values increased with storage but they were kept lower in samples containing extracts in comparison to control whereas viscosity was observed to higher in control (without extract) at the end of storage in citrus oil samples. The concentration of extract in the oil samples also showed partial changes in the studied parameters and the most profound effects were observed at highest extract concentration $(0.5 \%)$ used in this study.

\section{Acknowledgement}

The authors would like to extend their sincere appreciation to the Deanship of Scientific Research at King Saud University - Saudi Arabia for its funding this Research Group No (RG-1439-016).

\section{References}

1) Abu-Reidah, I.M.; Jamous, R.M.; Ali-Shtayeh, M.S. Phytochemistry, pharmacological properties and industrial applications of Rhus coriaria L. (Sumac). Jordan J. Biol.l Sci. 7, 233-244(2014).

2) Norton, S.; Lily, Y.B. trans- De materia medica by Pedanius Dioscorides. Hildesheim, Germany, Olms-Weidmann, 2005. 540 pp. J. History Med. Allied Sci. 61, 218-220 (2006).

3) Özcan, M. Effect of $\operatorname{sumach}$ (Rhus coriaria L.), extracts on the oxidative stability of peamut oil. J. Med. Food 6, 63-66 (2003).

4) Özcan, M. Antioxidant activities of rosemary, sage, and Sumac extracts and their combinations on stability of natural peanut oil. J. Med. Food 6, 267-270 (2003).

5) Özcan, M.; Haciseferogullari, H. A condiment[sumac (Rhus coriaria L.) fruits]: Some physicochemical properties. Bulg. J. Plant Physiol. 30(3-4), 74-84 (2004).

6) Rayne, S.; Mazza, G. Biological activities of extracts from Sumac (Rhus spp.): A review. Plant Foods Hum. Nutr. 62, 165-175 (2007).

7) Kossah, R.; Nsabimana, C.; Zhang, H.; Chen, W. Optimization of extraction of polyphenols from Syrian sumac (Rhus coriaria L.) and Chinese sumac (Rhus typhina L.) fruits. Res. J. Phytochem. 4, 146-153 (2010).

8) Al-Boushi, M.A.; Hamdo, H.H.; Herbali, J. Extraction and study of the phenolic compounds in the leaves and sticks of the Syrian sumac plant (Rhus coriaria L.) . Int. J. Chem. Technol. Res. 6, 2414-2420 (2014).

9) Kossah, R.; Nsabimana, C.; Zhang, H.; Chen, W. Evaluation of antimicrobial and antioxidant activities of Syrian Sumac fruit extract. J. Nat. Prod. 6, 96-102 (2013).

10) Candan, F.; Sökmen, A. Effects of Rhus coriaria L. (Anacardiaceae) on lipid peroxidation and free radical scavenging activity. Phytother. Res. 18, 84-86 (2004).

11) Özcan, M.; Akgul, A. Antioxidant activity of extracts and essential oils from Turkish spices on sunflower oil. Acta Aliment. Hung. 24, 81-90 (1995). 
12) Ferk, F.; Chakraborty, A.; Simic, T.; Kundi, M.; Knasmüller, S. Antioxidant and free radical scavenging activities of Sumac (Rhus coriaria) and identification of gallic acid as its active principle. BioMed. Central Pharmacol. 7, A71 (2007).

13) Bursal, E.; Köksal, E. Evaluation of reducing power and radical scavenging activities of water and ethanol extracts from sumac (Rhus coriaria L.). Food Res. Int. 44, 2217-2221 (2011).

14) Bozkurt, H. Investigation of the effect of Sumac extract and BHT addition on the quality of sucuk (Turkish dry-fermented sausage). J. Sci. Food Agric. 86, 849-856 (2006).

15) AOAC, Official Methods and recommended practices (Vol.1, 4th ed.). American Oil Chemists' Society, Champaign, IL (1990).

16) Püskülcü, H.; İkiz, F. Introduction to Statistic. Bilgehan Press. Bornova. İzmir, Turkey (1989). (in Turkish).

17) Kosar, M.; Bozan, B.; Temelli, F.; Baser, K.H.C. Antioxidant activity and phenolic composition of sumac (Rhus coriaria L.) extracts. Food Chem. 103, 952-959 (2007).

18) Mazaheri Tehrani, M.; Hesarinejad, M.A.; Razavi, S.M.A.; Mohamadian, R.; Poorkian, J. Comparing phy- sciochemical properties and antioxidant potential of sumac from Iran and Turkey. MOS Food Process. Technol. 5, 288-294(2017).

19) Al-Muwaly, K.Y.; Al-Flayeh, K.A.; Ali, A.A. Antioxidant and free radical scavenging effects of Iraqi sumac (Rhus coriaria L.). Baghdad Sci. J. 10, 921-933 (2013).

20) Wu, T.; McCallum, J.L.; Wang, S.; Liu, R.; Zhu, H.; Tsao, R. Evaluation of antioxidant activities and chemical characterisation of staghorn sumac fruit (Rhus hirta L.). Food Chem. 138, 1333-1340 (2013).

21) Ramadan, M.F.; Mörsel, J.T. Oxidative stability of black cumin (Nigella sativa L.), coriander(Coriandrum sativum L.) and niger (Guizotia abyssinica Cass.) crude seed oils upon stripping. Eur. J. Lipid Sci. Technol. 106, 35-43 (2004).

22) Mohdaly, A.A.A.; Sarhan, M.A.; Mahmoud, A.; Ramadan, M.F.; Smetanska, I. Antioxidant efficacy of potato peels and sugar beet pulp extracts in vegetable oils protection. Food Chem. 123, 1019-1026(2010).

23) Kiralan, M.; Ramadan, M.F. Volatile oxidation compounds and stability of safflower, sesame and canola cold-pressed oils as affected by thermal and microwave treatments. J. Oleo Sci. 65, 825-833 (2016). 